\title{
Prompt photon-jet angular correlations at central rapidities in $p+A$ collisions
}

\author{
Sanjin Benic ${ }^{1}$ and Adrian Dumitru ${ }^{2,3,4}$ \\ ${ }^{1}$ Physics Department, Faculty of Science, University of Zagreb, Zagreb 10000, Croatia \\ ${ }^{2}$ Department of Natural Sciences, Baruch College, CUNY, 17 Lexington Avenue, New York 10010, USA \\ ${ }^{3}$ The Graduate School and University Center, The City University of New York, \\ 365 Fifth Avenue, New York 10016, USA \\ ${ }^{4}$ Physics Department, Brookhaven National Lab, Upton, New York 11973, USA
}

(Received 10 October 2017; published 24 January 2018)

\begin{abstract}
Photon-jet azimuthal correlations in proton-nucleus collisions are a promising tool for gaining information on the gluon distribution of the nucleus in the regime of nonlinear color fields. We compute such correlations from the process $g \rightarrow q \bar{q} \gamma$ in the rapidity regime where both the projectile and target lightcone momentum fractions are small. By integrating over the phase space of the quark which emits the photon, subject to the restriction that the photon picks up most of the transverse momentum (to pass an isolation cut), we effectively obtain a $g+A \rightarrow q \gamma$ process. For nearly back-to-back photon-jet configurations we find that it dominates over the leading-order process $q+A \rightarrow q \gamma$ by two less powers of $Q_{\perp} / Q_{S}$, where $\boldsymbol{Q}_{\perp}$ and $Q_{S}$ denote the net photon-jet pair momentum and the saturation scale of the nucleus, respectively. We determine the transverse-momentum-dependent gluon distributions involved in $g+A \rightarrow$ $q \gamma$ and the scale where they are evaluated. Finally, we provide analytic expressions for $\langle\cos n \phi\rangle$ moments, where $\phi$ is the angle between $\boldsymbol{Q}_{\perp}$ and the average photon-jet transverse momentum $\tilde{\boldsymbol{P}}_{\perp}$, and first qualitative estimates of their transverse momentum dependence.
\end{abstract}

DOI: 10.1103/PhysRevD.97.014012

\section{INTRODUCTION}

Angular correlations between hadrons and prompt photons in high-energy collisions have been suggested to provide insight into the gluon fields of hadrons or nuclei in the small- $x$, nonlinear regime [1-6]. Prompt photons are those originating from hard interactions among the initial beam partons. Those studies have mostly focused on photon or dilepton production through the leading-order $q \rightarrow q \gamma$ process (in the field of the target) which dominates in the fragmentation region of the projectile at forward rapidities. In the central region on the other hand particle production is dominated by processes involving soft partons from both projectile and target. In particular, it has been pointed out in Ref. [7] that the production of a photon can also occur via $g \rightarrow q \bar{q} \gamma$. Although the amplitude squared for this process is suppressed by one additional power of $\alpha_{s}$ as compared to $q \rightarrow q \gamma$, on the other hand the gluon density in the projectile at small $x$ formally

Published by the American Physical Society under the terms of the Creative Commons Attribution 4.0 International license. Further distribution of this work must maintain attribution to the author(s) and the published article's title, journal citation, and DOI. Funded by SCOAP ${ }^{3}$. is of order $1 / \alpha_{s}$ times the density of quarks and so these contributions should be comparable.

Here we consider angular correlations between a photon with transverse momentum $\boldsymbol{k}_{\gamma \perp}$ and a jet with transverse momentum $\boldsymbol{p}_{\perp}$. We obtain this final state by integrating over the phase space of the quark from which the photon is emitted subject to the restriction that the photon takes most of the transverse momentum. The contribution from the quark to photon fragmentation is explicitly taken into account. Hence, our process becomes $g \rightarrow q \gamma$. We show that in the back-to-back correlation limit where the photonjet transverse momentum imbalance $\boldsymbol{Q}_{\perp}=\boldsymbol{p}_{\perp}+\boldsymbol{k}_{\gamma \perp}$ is much smaller than their average transverse momentum $\tilde{\boldsymbol{P}}_{\perp}=\left(\boldsymbol{p}_{\perp}-\boldsymbol{k}_{\gamma \perp}\right) / 2$, and smaller than the saturation scale $Q_{S}$ of the nucleus, the $g \rightarrow q \gamma$ process in fact dominates over $q \rightarrow q \gamma$ by two (fewer) powers of $Q_{\perp} / Q_{S}$.

The $q \rightarrow q \gamma$ process can be written in terms of a convolution of hard factors with the dipole forward scattering amplitude $[1-4,6] . g \rightarrow q \bar{q} \gamma$, on the other hand, in general involves the expectation value of a correlator of four Wilson lines at small $x$ (see below and Ref. [7]). In the near back-to-back limit $Q_{\perp}^{2} / \tilde{P}_{\perp}^{2} \ll 1$, however, this correlator can be expressed in terms of transverse-momentumdependent gluon distributions [8-10]. Below, we determine which gluon distributions appear in photon-jet production 
through the $g \rightarrow q \gamma$ process, and at which transverse momentum scale they are evaluated. This turns out to be given by the total transverse momentum in the final state; thus the process can be used to obtain information about the gluon distributions near the nonlinear "saturation" scale. Our focus here is on proton-nucleus $(p+A)$ collisions at high energies where the saturation scale $Q_{S}$ of the heavy ion is expected to be semihard, on the order of a few $\mathrm{GeV}$. We employ the "color glass condensate" formalism [11] to describe the strong yet weakly coupled gluon fields of the nucleus at small $x$. For completeness let us mention that photon-jet production in proton-proton $(p+p)$ collisions has been analyzed in the $k_{T}$-factorization approach with transverse-momentum-dependent gluon distributions in Refs. [12]. References $[13,14]$ considered photon-jet production in collinear factorization at NLO supplemented by a parton shower generator. Lastly, such angular correlations from $q \bar{q} \rightarrow \gamma g$ annihilation in $p \bar{p}$ collisions have been discussed in Ref. [15]. Our main interest here is to show how photon-jet azimuthal correlations at small $x$ probe the gluon fields of the heavy-ion target in the nonlinear regime.

From the cross section for $g \rightarrow q \gamma$ we can compute $\langle\cos n \phi\rangle$ angular moments, where $\phi$ denotes the angle between $\boldsymbol{Q}_{\perp}$ and $\tilde{\boldsymbol{P}}_{\perp}$. These are nonzero for all $n$ but increasingly suppressed as $\left(Q_{\perp} / \tilde{P}_{\perp}\right)^{n}$. The transverse momentum dependence of these moments in protonnucleus collisions provides information about the gluon distributions of the heavy-ion target. We also compute the "azimuthal harmonics" $\langle\cos n \Phi\rangle(n=1 \ldots 3)$ which are frequently studied in heavy-ion collisions, where now $\Phi$ is the angle between $\boldsymbol{k}_{\gamma \perp}$ and $\boldsymbol{p}_{\perp}$.

Photon-hadron (or jet) correlations have been studied experimentally by the PHENIX [16] and STAR collaborations [17] at the BNL-RHIC accelerator, and by CMS and ATLAS at the CERN-LHC. Thus far the experiments at RHIC have focused on $p+p$ or nucleus-nucleus $(A+A)$ collisions. The latter provide insight mainly into final state interactions of the jet with the hot and dense medium produced in heavy-ion collisions. Photon-jet correlations in $p+p$ collisions have provided information on transversemomentum-dependent gluon distributions albeit not in the weakly coupled, nonlinear regime of QCD since the saturation momentum of a proton at RHIC energies (on average over impact parameters) is well below $1 \mathrm{GeV}$ [18].

The ATLAS Collaboration has measured photon-jet correlations in $p+p$ collisions at $7 \mathrm{TeV}$ [19]. For $p_{\perp}^{\mathrm{jet}}>$ $40 \mathrm{GeV}$ and $E_{\perp}^{\gamma}>45 \mathrm{GeV}$ the correlations near $\phi=\pi$ can be described reasonably well by established QCD Monte Carlo generators. The CMS Collaboration, too, has analyzed these correlations in $p+p$ and $A+A$ collisions but also obtained data for $p+P b$ collisions at $5 \mathrm{TeV}$ [20]. Interestingly, for $p_{\perp}^{\text {jet }}>30 \mathrm{GeV}$ and $40 \mathrm{GeV}<$ $k_{\perp}^{\gamma}<50 \mathrm{GeV}$ the correlation strength near $\phi=\pi$ appears to be overestimated by some Monte Carlo models; see also Fig. 6 in Refs. [14]. This may potentially be related to high gluon density effects of the kind considered here, especially if confirmed by more symmetric configurations with smaller $Q_{\perp}$ (say, by going to lower $k_{\perp}^{\gamma}>30 \mathrm{GeV}$ ).

This paper is organized as follows. In Sec. II we recall the leading-order (LO) cross section expressed in terms of the transverse momentum imbalance $\boldsymbol{Q}_{\perp}$ and the average photon-jet transverse momentum $\tilde{\boldsymbol{P}}_{\perp}$. The NLO amplitude is introduced in Sec. III, and expanded in powers of gluon momenta in the follow-up Appendix A. In Sec. IV we obtain the cross section for $g \rightarrow q \bar{q} \gamma$, while in Appendix B we compute the nuclear distribution functions appearing in the next-to-leading-order (NLO) cross section. The $g \rightarrow q \gamma$ cross section and the angular moments $a_{n}$ are obtained in Sec. V B. Our results are summarized in Sec. VI.

\section{LEADING-ORDER PHOTON-JET CROSS SECTION}

The LO photon-jet cross section due to scattering of an (anti)quark off the target is given by [2-5]

$$
\begin{aligned}
& \frac{\mathrm{d} \sigma}{\mathrm{d}^{2} \boldsymbol{k}_{\gamma \perp} \mathrm{d} \eta_{k_{\gamma}} \mathrm{d}^{2} \boldsymbol{p}_{\perp} \mathrm{d} \eta_{p}} \\
& =\frac{\alpha_{e} q_{f}^{2}}{32 \pi^{5}} \int \mathrm{d} x_{p}\left[f_{q, p}\left(x_{p}, Q^{2}\right)+f_{\bar{q}, p}\left(x_{p}, Q^{2}\right)\right] \\
& \quad \times \frac{1}{l^{+}}\left(l^{+2}+p^{+2}\right)\left[\frac{l \cdot p}{\left(l \cdot k_{\gamma}\right)\left(p \cdot k_{\gamma}\right)}+\frac{1}{p \cdot k_{\gamma}}-\frac{1}{l \cdot k_{\gamma}}\right] \\
& \quad \times \mathcal{N}_{F}\left(x_{A},\left(\boldsymbol{p}_{\perp}+\boldsymbol{k}_{\gamma \perp}\right)^{2}\right)(2 \pi) \delta\left(l^{+}-p^{+}-k_{\gamma}^{+}\right),
\end{aligned}
$$

where $k_{\gamma}$ and $p$ are the photon and jet momenta, respectively, while $l$ is the momentum of the quark from the proton; see Fig. 1. Here $f_{q(\bar{q}), p}\left(x_{p}, Q^{2}\right)$ denote the collinear quark and the antiquark distribution functions of the proton evaluated at some hard scale $Q^{2}$ and $\mathcal{N}_{F}\left(x_{A}, k_{\perp}^{2}\right)$ is the forward scattering amplitude of a fundamental dipole off the nucleus. At $x_{A} \ll 1$ and in the large- $N_{c}$ limit this is obtained by solving the Balitsky-Kovchegov (BK) evolution equation [21].

We can rewrite the cross section in terms of the light-cone momentum fraction of the outgoing quark $z_{q}=p^{+} / l^{+}$and the transverse momentum imbalance

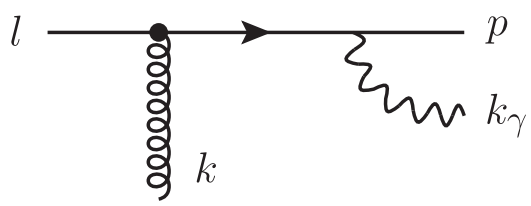

FIG. 1. Example for a LO diagram for photon production in the high-energy limit. The blob contains multiple (eikonal) scatterings on the dense target with momentum exchange $k$. The complete LO contribution includes photon emission before the interaction with the nucleus (not shown). 
$\boldsymbol{Q}_{\perp}$ and the average transverse momentum $\tilde{\boldsymbol{P}}_{\perp}$ which are defined via

$$
\boldsymbol{Q}_{\perp} \equiv \boldsymbol{k}_{\gamma \perp}+\boldsymbol{p}_{\perp}, \quad \tilde{\boldsymbol{P}}_{\perp} \equiv \frac{1}{2}\left(\boldsymbol{p}_{\perp}-\boldsymbol{k}_{\gamma \perp}\right) .
$$

In terms of these variables,

$$
\begin{aligned}
& \frac{\mathrm{d} \sigma}{\mathrm{d}^{2} \tilde{\boldsymbol{P}}_{\perp} \mathrm{d}^{2} \boldsymbol{Q}_{\perp} \mathrm{d} \eta_{k_{\gamma}} \mathrm{d} \eta_{p}} \\
& =\frac{\alpha_{e} q_{f}^{2}}{16 \pi^{5}} \int \mathrm{d} x_{p}\left[f_{q, f}\left(x_{p}, Q^{2}\right)+f_{\bar{q}, f}\left(x_{p}, Q^{2}\right)\right] \\
& \quad \times \frac{1+z_{q}^{2}}{z_{q}} \frac{z_{q}\left(1-z_{q}\right)^{2} Q_{\perp}^{2}}{\left(\frac{1}{2} \boldsymbol{Q}_{\perp}-\tilde{\boldsymbol{P}}_{\perp}\right)^{2}\left[\left(\frac{1}{2}-z_{q}\right) \boldsymbol{Q}_{\perp}+\tilde{\boldsymbol{P}}_{\perp}\right]^{2}} \\
& \quad \times \mathcal{N}_{F}\left(x_{A}, Q_{\perp}^{2}\right)(2 \pi) p^{+} \delta\left(l^{+}-p^{+}-k_{\gamma}^{+}\right) .
\end{aligned}
$$

We focus on photon-jet configurations that correspond to $\tilde{P}_{\perp} \gg Q_{S} \gg Q_{\perp}$ so that the transverse momenta of the photon and the jet are almost back to back. In this limit, due to nonlinear effects the dipole forward scattering amplitude $\mathcal{N}_{F}\left(x_{A}, Q_{\perp}^{2}\right) \sim 1 / Q_{S}^{2}\left(x_{A}\right)$ is proportional to the inverse saturation scale squared of the nucleus. Hence, the leading contribution to the LO cross section is of order $\left(Q_{\perp} / Q_{S}\right)^{2}$, times an overall $1 / \tilde{P}_{\perp}^{4}$, and is independent of the angle $\phi$ between $\boldsymbol{Q}_{\perp}$ and $\tilde{\boldsymbol{P}}_{\perp}$. An angular dependence $\sim \cos \phi$ arises at next-to-leading power $\left(Q_{\perp} / Q_{S}\right)^{2} \boldsymbol{Q}_{\perp} \cdot \tilde{\boldsymbol{P}}_{\perp} / \tilde{\boldsymbol{P}}_{\perp}^{2}$. Below we show that the cross section for $g \rightarrow q \gamma$ generates an isotropic contribution at order 1 (times the common $\left.1 / \tilde{P}_{\perp}^{4}\right)$, a $\cos \phi$ angular dependence already at next-toleading power $\sim Q_{\perp} / \tilde{P}_{\perp}$, and so on.

\section{NEXT-TO-LEADING-ORDER PHOTON-JET AMPLITUDE}

At the NLO order photons are produced via the $g \rightarrow q \bar{q} \gamma$ process where either the incoming gluon or either of the quarks may scatter off the field of the target; see Fig. 2. For a photon-jet final state, one of the quarks will eventually be integrated over. ${ }^{1}$ The external momenta in the process are $\boldsymbol{q}$, $\boldsymbol{p}$ and $\boldsymbol{k}_{\gamma}$ for the quark, antiquark and the photon, respectively. In the amplitude we also have the transverse gluon momenta from the proton, $\boldsymbol{k}_{1 \perp}$, and from the nucleus, $\boldsymbol{k}_{\perp}$ and $\boldsymbol{k}_{2 \perp}-\boldsymbol{k}_{\perp}$ (there are two, because of the two Wilson lines). Here, $\boldsymbol{k}_{2 \perp} \equiv \boldsymbol{P}_{\perp}-\boldsymbol{k}_{1 \perp}$ with $\boldsymbol{P}_{\perp} \equiv \boldsymbol{q}_{\perp}+\boldsymbol{p}_{\perp}+\boldsymbol{k}_{\gamma \perp}$ is the total final state transverse momentum.

The main formula for the amplitude is [see Eq. (47) in [7]]

\footnotetext{
${ }^{1}$ Specifically, we integrate over the phase space of the quark which emits the photon. We restrict to the region of phase space where the photon picks up most of the momentum of the parent quark so that the configuration passes a photon isolation cut; see Sec. V below.
}
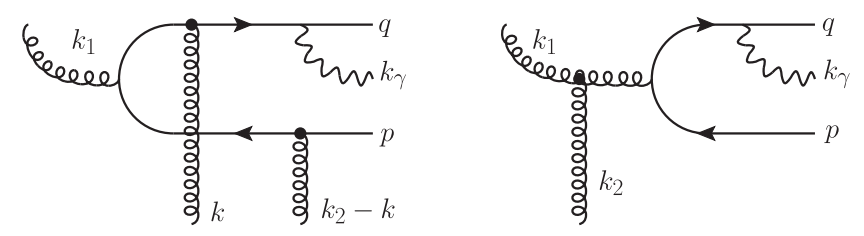

FIG. 2. Examples for NLO diagrams for photon production in the high-energy limit. In the left diagram the gluon from the proton emits a $q \bar{q}$ pair which then scatters on the dense nucleus. In the right diagram the gluon scatters on the nucleus and then produces a $q \bar{q}$ pair and a photon. The complete NLO contribution includes diagrams where the photon is emitted from the antiquark line, or from a virtual quark-antiquark state (not shown) in the case of single-inclusive photon production.

$$
\begin{aligned}
\mathcal{M}^{\mu}\left(\boldsymbol{p}, \boldsymbol{q}, \boldsymbol{k}_{\gamma}\right) & \\
= & -q_{f} e g^{2} \int_{\boldsymbol{k}_{\perp} \boldsymbol{k}_{\perp \perp}} \int_{\boldsymbol{x}_{\perp} \boldsymbol{y}_{\perp}} \frac{\rho_{p}^{a}\left(\boldsymbol{k}_{1 \perp}\right)}{k_{1 \perp}^{2}} e^{i \boldsymbol{k}_{\perp} \cdot \boldsymbol{x}_{\perp}+i\left(\boldsymbol{k}_{\perp \perp}-\boldsymbol{k}_{\perp}\right) \cdot \boldsymbol{y}_{\perp}} \\
& \times \bar{u}(\boldsymbol{q})\left\{T_{g}^{\mu}\left(\boldsymbol{k}_{1 \perp}\right) U\left(\boldsymbol{x}_{\perp}\right)^{b a} t^{b}\right. \\
& \left.+T_{q \bar{q}}^{\mu}\left(\boldsymbol{k}_{\perp}, \boldsymbol{k}_{1 \perp}\right) \tilde{U}\left(\boldsymbol{x}_{\perp}\right) t^{a} \tilde{U}^{\dagger}\left(\boldsymbol{y}_{\perp}\right)\right\} v(\boldsymbol{p}) .
\end{aligned}
$$

Here $\tilde{U}\left(\boldsymbol{x}_{\perp}\right)\left[U\left(\boldsymbol{x}_{\perp}\right)\right]$ is the Wilson line in the fundamental (adjoint) representation

$$
\begin{aligned}
\tilde{U}\left(\boldsymbol{x}_{\perp}\right) & \equiv \mathcal{P}_{+} \exp \left[-i g^{2} \int_{-\infty}^{\infty} d z^{+} \frac{1}{\boldsymbol{\nabla}_{\perp}^{2}} \rho_{A}^{a}\left(z^{+}, \boldsymbol{x}_{\perp}\right) t^{a}\right], \\
U\left(\boldsymbol{x}_{\perp}\right) & \equiv \mathcal{P}_{+} \exp \left[-i g^{2} \int_{-\infty}^{\infty} d z^{+} \frac{1}{\boldsymbol{\nabla}_{\perp}^{2}} \rho_{A}^{a}\left(z^{+}, \boldsymbol{x}_{\perp}\right) T^{a}\right],
\end{aligned}
$$

with $t^{a}\left(T^{a}\right)$ the $S U\left(N_{c}\right)$ generators in the fundamental (adjoint) representation. $g \rho_{p}^{a}\left(\boldsymbol{x}_{\perp}\right)\left[g \rho_{A}^{a}\left(\boldsymbol{x}_{\perp}\right)\right]$ denotes the random color charge density of the proton (nucleus) which will be averaged over after squaring the amplitude. The other factor of the coupling $g$ in Eq. (4) arises from the $g-q-\bar{q}$ vertex.

We also introduce the following momentum labels,

$$
\begin{aligned}
& w \equiv q+k_{\gamma}, \quad v \equiv p+k_{\gamma}, \quad g \equiv q-k, \quad h \equiv q-k-k_{1}, \\
& u \equiv q+k_{\gamma}-k, \quad l \equiv q+k_{\gamma}-k-k_{1},
\end{aligned}
$$

where $k^{+}=0, k_{1}^{-}=0, k_{1}^{+}=P^{+}$. We also define the Lipatov vertex

$$
\begin{aligned}
C_{L}^{+}\left(q, \boldsymbol{k}_{1 \perp}\right) & =q^{+}-\frac{k_{1 \perp}^{2}}{q^{-}+i \epsilon}, \\
C_{L}^{-}\left(q, \boldsymbol{k}_{1 \perp}\right) & =\frac{\left(\boldsymbol{q}_{\perp}-\boldsymbol{k}_{1 \perp}\right)^{2}}{q^{+}}-q^{-}, \\
\boldsymbol{C}_{L \perp}\left(q, \boldsymbol{k}_{1 \perp}\right) & =\boldsymbol{q}_{\perp}-2 \boldsymbol{k}_{1 \perp} .
\end{aligned}
$$

We then have 


$$
T_{g}^{\mu} \equiv \sum_{\beta=1}^{2} R_{\beta}^{\mu}, \quad T_{q \bar{q}}^{\mu} \equiv \sum_{\beta=9}^{12} R_{\beta}^{\mu},
$$

with

$$
\begin{aligned}
& R_{1}^{\mu}=-\frac{\gamma^{\mu}(\not w+m) \mathscr{C}_{L}}{P^{2}\left(w^{2}-m^{2}\right)}, \quad R_{2}^{\mu}=-\frac{\varnothing_{L}(-\not p+m) \gamma^{\mu}}{P^{2}\left(v^{2}-m^{2}\right)}, \\
& R_{9}^{\mu}=-\frac{\gamma^{\mu}(\not w+m) \gamma^{+}(\not h+m) \gamma^{-}(\not l+m) \gamma^{+}}{N_{k}\left(w^{2}-m^{2}\right)}, \\
& N_{k}=2 p^{+} M^{2}\left(u_{\perp}\right)+2 w^{+} M^{2}\left(l_{\perp}\right), \\
& R_{10}^{\mu}=-\frac{\gamma^{+}(\not g+m) \gamma^{-}(\not h+m) \gamma^{+}(-\not \gamma+m) \gamma^{\mu}}{N_{q}\left(v^{2}-m^{2}\right)}, \\
& N_{q} \equiv 2 v^{+} M^{2}\left(g_{\perp}\right)+2 q^{+} M^{2}\left(h_{\perp}\right) \text {, } \\
& R_{11}^{\mu}=2 p^{+} \frac{\gamma^{+}(\not g+m) \gamma^{\mu}(\not h+m) \gamma^{-}(\not l+m) \gamma^{+}}{N_{k} S}, \\
& k^{-}=w^{-}+\frac{M^{2}\left(\boldsymbol{l}_{\perp}\right)}{2 p^{+}}, \\
& R_{12}^{\mu}=2 q^{+} \frac{\gamma^{+}(\not g+m) \gamma^{-}(\not h+m) \gamma^{\mu}(\not l+m) \gamma^{+}}{N_{q} S}, \\
& k^{-}=q^{-}-\frac{M^{2}\left(g_{\perp}\right)}{2 q^{+}},
\end{aligned}
$$

with

$$
S=4 p^{+} q^{+} k_{\gamma}^{-}+2 q^{+} M^{2}\left(l_{\perp}\right)+2 p^{+} M^{2}\left(g_{\perp}\right) .
$$

Here we defined the transverse mass function $M\left(k_{\perp}\right) \equiv$ $\sqrt{k_{\perp}^{2}+m^{2}}$.

We assume that there is a certain combination of the external momenta which is the largest scale in the process (i.e. the average transverse momentum $\tilde{\boldsymbol{P}}_{\perp}$ of photon and jet). This scale is also supposed to be much larger than the saturation scale of the proton or that of the nucleus. The transverse momenta of the gluon from the proton, $k_{1 \perp}$, and of the gluons from the nucleus, $k_{\perp}$ and $\left|\boldsymbol{k}_{2 \perp}-\boldsymbol{k}_{\perp}\right|$, are of the order of the respective saturation scales, much smaller than the hard scale.

With this assumption we perform an expansion of the amplitude in $\boldsymbol{k}_{1 \perp}, \boldsymbol{k}_{2 \perp}-\boldsymbol{k}_{\perp}$ and $\boldsymbol{k}_{\perp}$. To obtain the gluon distributions we must expand to first order in these momenta. The main point is that by Ward identities this expansion necessarily involves terms proportional to $k_{1 \perp}^{i}\left(k_{2}-k_{\perp}\right)^{j}$ or $k_{1 \perp}^{i} k_{\perp}^{j}$ in the amplitude. Note that by conservation of momentum this implies that the total transverse momentum $\boldsymbol{P}_{\perp} \equiv \boldsymbol{q}_{\perp}+\boldsymbol{p}_{\perp}+\boldsymbol{k}_{\gamma \perp}$ in the final state is much smaller than the average transverse momentum $\tilde{\boldsymbol{P}}_{\perp}$ of the photon and jet. We shall return to this point in Sec. V B. After some algebra (the full details are given in Appendix A), we obtain the following main result for the amplitude:

$$
\begin{aligned}
& \mathcal{M}^{\mu}\left(\boldsymbol{p}, \boldsymbol{q}, \boldsymbol{k}_{\gamma}\right) \\
& =-q_{f} e g^{2} \int_{\boldsymbol{k}_{\perp} \boldsymbol{k}_{1 \perp}} \int_{\boldsymbol{x}_{\perp} \boldsymbol{y}_{\perp}} \frac{\rho_{p}^{a}\left(\boldsymbol{k}_{1 \perp}\right)}{k_{1 \perp}^{2}} e^{i \boldsymbol{k}_{\perp} \cdot \boldsymbol{x}_{\perp}+i\left(\boldsymbol{P}_{\perp}-\boldsymbol{k}_{\perp}-\boldsymbol{k}_{\perp \perp}\right) \cdot \boldsymbol{y}_{\perp}} \\
& \quad \times \bar{u}(\boldsymbol{q})\left\{k_{2 i} R_{g}^{\mu i} U\left(\boldsymbol{x}_{\perp}\right)^{b a} t^{b}+\left[k_{i} R_{q}^{\mu i}+\left(k_{2}-k\right)_{i} R_{\bar{q}}^{\mu i}\right]\right. \\
& \left.\quad \times \tilde{U}\left(\boldsymbol{x}_{\perp}\right) t^{a} \tilde{U}^{\dagger}\left(\boldsymbol{y}_{\perp}\right)\right\} v(\boldsymbol{p}),
\end{aligned}
$$

where we defined

$$
R_{g}^{\mu i} \equiv \sum_{\beta=1}^{2} R_{\beta}^{\mu i}, \quad R_{q}^{\mu i} \equiv \sum_{\beta=3}^{5} R_{\beta}^{\mu i}, \quad R_{\bar{q}}^{\mu i} \equiv \sum_{\beta=6}^{8} R_{\beta}^{\mu i},
$$

with

$R_{1}^{\mu i} \equiv \frac{2}{P^{2}} \gamma^{\mu} \frac{\not 1+\not k_{\gamma}+m}{\left(q+k_{\gamma}\right)^{2}-m^{2}} \not k_{2} \frac{k_{1}^{i}}{P^{+} P^{-}}$,

$R_{2}^{\mu i} \equiv \frac{2}{P^{2}} \not k_{2} \frac{-\not p-\not k_{\gamma}+m}{\left(p+k_{\gamma}\right)^{2}-m^{2}} \gamma^{\mu} \frac{k_{1}^{i}}{P^{+} P^{-}}$,

$R_{3}^{\mu i} \equiv-\gamma^{\mu} \frac{\not 1+\not k_{\gamma}+m}{\left(q+k_{\gamma}\right)^{2}-m^{2}} \frac{\gamma^{i}}{P^{-}} \frac{\not k_{1}-\not p+m}{\left(k_{1}-p\right)^{2}-m^{2}} \frac{\not k_{1 \perp}}{P^{+}}$,

$R_{4}^{\mu i} \equiv-\frac{\gamma^{i}}{P^{-}} \frac{\not 1-\not k_{2}+m}{\left(q-k_{2}\right)^{2}-m^{2}} \gamma^{\mu} \frac{\not k_{1}-\not p+m}{\left(k_{1}-p\right)^{2}-m^{2}} \frac{\not k_{1 \perp}}{P^{+}}$,

$R_{5}^{\mu i} \equiv-\frac{\gamma^{i}}{P^{-}} \frac{\not 1-\not k_{2}+m}{\left(q-k_{2}\right)^{2}-m^{2}} \frac{\not k_{1 \perp}}{P^{+}} \frac{-\not p-\not k_{\gamma}+m}{\left(p+k_{\gamma}\right)^{2}-m^{2}} \gamma^{\mu}$,

$R_{6}^{\mu i} \equiv \frac{\not k_{1 \perp}}{P^{+}} \frac{\not 1-\not k_{1}+m}{\left(q-k_{1}\right)^{2}-m^{2}} \frac{\gamma^{i}}{P^{-}} \frac{-\not p-\not k_{\gamma}+m}{\left(p+k_{\gamma}\right)^{2}-m^{2}} \gamma^{\mu}$,

$R_{7}^{\mu i} \equiv \frac{\not k_{1 \perp}}{P^{+}} \frac{\not 1-\not k_{1}+m}{\left(q-k_{1}\right)^{2}-m^{2}} \gamma^{\mu} \frac{\not k_{2}-\not p+m}{\left(k_{2}-p\right)^{2}-m^{2}} \frac{\gamma^{i}}{P^{-}}$,

$R_{8}^{\mu i} \equiv \gamma^{\mu} \frac{\not 1+\not k_{\gamma}+m}{\left(q+k_{\gamma}\right)^{2}-m^{2}} \frac{\not k_{1 \perp}}{P^{+}} \frac{\not k_{2}-\not p+m}{\left(k_{2}-p\right)^{2}-m^{2}} \frac{\gamma^{i}}{P^{-}}$.

Here $R_{\beta}^{\mu i}$, with $\beta=3, \ldots 5(\beta=6, \ldots, 8)$, correspond to the three terms in the first (second) line of Eq. (A10), after using the momentum definitions Eq. (6) and the collinear limit:

$$
\begin{aligned}
& k_{1}=\left(k_{1}^{+}, k_{1}^{-}, \boldsymbol{k}_{1 \perp}\right)=\left(P^{+}, 0,0\right), \\
& k_{2}=\left(k_{2}^{+}, k_{2}^{-}, \boldsymbol{k}_{2 \perp}\right)=\left(0, P^{-}, 0\right) .
\end{aligned}
$$

As an aside, it is useful to check the soft photon limit of the amplitude in Eq. (14). We obtain

$$
\begin{aligned}
\mathcal{M}^{\mu}\left(\boldsymbol{p}, \boldsymbol{q}, \boldsymbol{k}_{\gamma}\right)= & q_{f} e\left(\frac{p^{\mu}}{p \cdot k_{\gamma}}-\frac{q^{\mu}}{q \cdot k_{\gamma}}\right) g^{2} \\
& \times \int_{\boldsymbol{k}_{\perp} \boldsymbol{k}_{\perp \perp}} \int_{\boldsymbol{x}_{\perp} \boldsymbol{y}_{\perp}} \frac{\rho_{p}^{a}\left(\boldsymbol{k}_{1 \perp}\right)}{k_{1 \perp}^{2}} e^{i \boldsymbol{k}_{\perp} \cdot \boldsymbol{x}_{\perp}+i\left(\boldsymbol{P}_{\perp}-\boldsymbol{k}_{\perp}-\boldsymbol{k}_{\perp \perp}\right) \cdot \boldsymbol{y}_{\perp}} \\
& \times \bar{u}(\boldsymbol{q})\left\{k_{2 i} R_{g}^{i} U\left(\boldsymbol{x}_{\perp}\right)^{b a} t^{b}\right. \\
& \left.+\left[k_{i} R_{q}^{i}+\left(k_{2}-k\right)_{i} R_{\bar{q}}^{i}\right] \tilde{U}\left(\boldsymbol{x}_{\perp}\right) t^{a} \tilde{U}^{\dagger}\left(\boldsymbol{y}_{\perp}\right)\right\} v(\boldsymbol{p}),
\end{aligned}
$$


where

$$
\begin{aligned}
R_{g}^{i} & \equiv-\frac{2}{P^{2}} \not k_{2} \frac{k_{1}^{i}}{P^{+} P^{-}}, \\
R_{q}^{i} & \equiv \frac{\gamma^{i}}{P^{-}} \frac{\not k_{1}-\not p+m}{\left(k_{1}-p\right)^{2}-m^{2}} \frac{\not k_{1 \perp}}{P^{+}}, \\
R_{\bar{q}}^{i} & \equiv-\frac{\not l_{1 \perp}}{P^{+}} \frac{\not k_{2}-\not p+m}{\left(k_{2}-p\right)^{2}-m^{2}} \frac{\gamma^{i}}{P^{-}} .
\end{aligned}
$$

Thus, Eq. (18) factorizes into the soft photon emission amplitude times the amplitude for $q \bar{q}$ production. The $q \bar{q}$ production part exactly agrees with the result in Ref. [10] once the expressions in Eqs. (25) and (26) of [10] are compared with Eq. (19) through $R_{g, i}=\tilde{T}_{g, i}, R_{q, i}=\tilde{T}_{q \bar{q}, i}^{A}$ and
$R_{\bar{q}, i}=\tilde{T}_{q \bar{q}, i}^{B}$, together with $k_{1} \rightarrow k_{2}$ and $k_{2} \rightarrow k_{1}$, in the notation of Ref. [10].

\section{NEXT-TO-LEADING-ORDER CROSS SECTION}

We can immediately derive the cross section by adapting the calculation from Ref. [7]. With the replacements

$$
k_{2 i} R_{g}^{\mu i} \rightarrow T_{g}^{\mu}, \quad k_{i} R_{q}^{\mu i}+\left(k_{2}-k\right)_{i} R_{\bar{q}}^{\mu i} \rightarrow T_{q \bar{q}}^{\mu},
$$

the amplitude in Eq. (14) has the same structure as (4) [or Eq. (47) in [7]]. Then, we can deduce the cross section from Eq. (63) in [7]:

$$
\begin{aligned}
& \frac{d \sigma}{d^{2} \boldsymbol{p}_{\perp} d^{2} \boldsymbol{q}_{\perp} d^{2} \boldsymbol{k}_{\gamma \perp} d \eta_{p} d \eta_{q} d \eta_{k_{\gamma}}}=\frac{\alpha_{e} \alpha_{S} q_{f}^{2}}{256 \pi^{8} N_{c}\left(N_{c}^{2}-1\right)} \int_{\boldsymbol{k}_{1 \perp} \boldsymbol{k}_{2 \perp}}(2 \pi)^{2} \delta^{(2)}\left(\boldsymbol{P}_{\perp}-\boldsymbol{k}_{1 \perp}-\boldsymbol{k}_{2 \perp}\right) \frac{\varphi_{p}\left(x_{p}, k_{1 \perp}^{2}\right)}{k_{1 \perp}^{2}} \\
& \times \int_{\boldsymbol{x}_{\perp} \boldsymbol{x}_{\perp}^{\prime} \boldsymbol{y}_{\perp} \boldsymbol{y}_{\perp}^{\prime}} \int_{\boldsymbol{k}_{\perp}, \boldsymbol{k}_{\perp}^{\prime}} e^{i\left(\boldsymbol{k}_{\perp} \cdot \boldsymbol{x}_{\perp}-\boldsymbol{k}_{\perp}^{\prime} \cdot \boldsymbol{x}_{\perp}^{\prime}\right)+i\left(\boldsymbol{k}_{2 \perp}-\boldsymbol{k}_{\perp}\right) \cdot \boldsymbol{y}_{\perp}-i\left(\boldsymbol{k}_{2 \perp}-\boldsymbol{k}_{\perp}^{\prime}\right) \cdot \boldsymbol{y}_{\perp}^{\prime}} g^{\mu \mu^{\prime}} \\
& \times\left\{\operatorname{tr}\left[(\not d+m) k_{2}^{i} R_{g, \mu i}(m-\not p) \gamma^{0} k_{2}^{i^{\prime}} R_{g, \mu^{\prime} i^{\prime}}^{\prime \dagger}\right] C\left(x_{A}, \boldsymbol{x}_{\perp}, \boldsymbol{x}_{\perp}, \boldsymbol{x}_{\perp}^{\prime}, \boldsymbol{x}_{\perp}^{\prime}\right)\right. \\
& +\operatorname{tr}\left[(\not l+m) k_{2}^{i} R_{g, \mu i}(m-\not p) \gamma^{0}\left(k^{\prime i^{\prime}} R_{q, \mu^{\prime} i^{\prime}}^{\prime \dagger}+\left(k_{2}-k^{\prime}\right)^{i^{\prime}} R_{\bar{q}, \mu^{\prime} i^{\prime}}^{\prime \dagger}\right) \gamma^{0}\right] C\left(x_{A}, \boldsymbol{x}_{\perp}, \boldsymbol{x}_{\perp}, \boldsymbol{y}_{\perp}^{\prime}, \boldsymbol{x}_{\perp}^{\prime}\right) \\
& +\operatorname{tr}\left[(\not 1+m)\left(k^{i} R_{q, \mu i}+\left(k_{2}-k\right)^{i} R_{\bar{q}, \mu i}\right)(m-\not p) \gamma^{0} k_{2}^{i^{\prime}} R_{g, \mu^{\prime} i^{\prime}}^{\prime} \gamma^{0}\right] C\left(x_{A}, \boldsymbol{x}_{\perp}, \boldsymbol{y}_{\perp}, \boldsymbol{x}_{\perp}^{\prime}, \boldsymbol{x}_{\perp}^{\prime}\right) \\
& +\operatorname{tr}\left[(\not q+m)\left(k^{i} R_{q, \mu i}+\left(k_{2}-k\right)^{i} R_{\bar{q}, \mu i}\right)(m-\not p) \gamma^{0}\left(k^{\prime i^{\prime}} R_{q, \mu^{\prime} i^{\prime}}^{\prime \dagger}+\left(k_{2}-k^{\prime}\right)^{i^{\prime}} R_{\bar{q}, \mu^{\prime} i^{\prime}}^{\dagger}\right) \gamma^{0}\right] \\
& \left.\times C\left(x_{A}, \boldsymbol{x}_{\perp}, \boldsymbol{y}_{\perp}, \boldsymbol{y}_{\perp}^{\prime}, \boldsymbol{x}_{\perp}^{\prime}\right)\right\} \text {. }
\end{aligned}
$$

To follow more closely the notation in [10] we rewrote the nuclear gluon field correlators from Eqs. (58)-(60) in Ref. [7] in terms of the four Wilson line correlator

$$
C\left(x_{A}, \boldsymbol{x}_{\perp}, \boldsymbol{y}_{\perp}, \boldsymbol{y}_{\perp}^{\prime}, \boldsymbol{x}_{\perp}^{\prime}\right) \equiv \operatorname{tr}_{c}\left\langle\tilde{U}\left(\boldsymbol{x}_{\perp}\right) t^{a} \tilde{U}^{\dagger}\left(\boldsymbol{y}_{\perp}\right) \tilde{U}\left(\boldsymbol{y}_{\perp}^{\prime}\right) t^{a} \tilde{U}^{\dagger}\left(\boldsymbol{x}_{\perp}^{\prime}\right)\right\rangle_{x_{A}} .
$$

We used $t^{b} U^{b a}\left(\boldsymbol{x}_{\perp}\right)=\tilde{U}\left(\boldsymbol{x}_{\perp}\right) t^{a} \tilde{U}\left(\boldsymbol{x}_{\perp}\right)$. Also, we defined the unintegrated gluon distribution $\varphi_{p}\left(x_{p}, k_{1 \perp}^{2}\right)$ of the proton via

$$
\left\langle\rho_{p}^{a}\left(\boldsymbol{k}_{1 \perp}\right) \rho_{p}^{\dagger b}\left(\boldsymbol{k}_{1 \perp}\right)\right\rangle_{x_{p}} \equiv \frac{\delta^{a b} k_{1 \perp}^{2}}{2 \pi N_{\mathrm{c}} C_{F} g^{2}} \varphi_{p}\left(x_{p}, k_{1 \perp}^{2}\right) .
$$

The proton and the nucleus light-cone momentum fractions, $x_{p}$ and $x_{A}$, are related to the final state kinematics via

$$
\begin{aligned}
& x_{p} \sqrt{\frac{s}{2}}=p^{+}+k_{\gamma}^{+}+q^{+}, \\
& x_{A} \sqrt{\frac{s}{2}}=p^{-}+k_{\gamma}^{-}+q^{-} .
\end{aligned}
$$

By partial integration we can turn powers of $k^{i},\left(k_{2}-k\right)^{i}$ and $k_{2}^{i}$ into gradients of the correlator of four Wilson lines (22). For example, 


$$
\begin{aligned}
& \int_{\boldsymbol{x}_{\perp} \boldsymbol{x}_{\perp}^{\prime} \boldsymbol{y}_{\perp} \boldsymbol{y}_{\perp}^{\prime}} \int_{\boldsymbol{k}_{\perp}, \boldsymbol{k}_{\perp}^{\prime}} e^{i\left(\boldsymbol{k}_{\perp} \cdot \boldsymbol{x}_{\perp}-\boldsymbol{k}_{\perp}^{\prime} \cdot \boldsymbol{x}_{\perp}^{\prime}\right)+i\left(\boldsymbol{k}_{\perp \perp}-\boldsymbol{k}_{\perp}\right) \cdot \boldsymbol{y}_{\perp}-i\left(\boldsymbol{k}_{2 \perp}-\boldsymbol{k}_{\perp}^{\prime}\right) \cdot \boldsymbol{y}_{\perp}^{\prime}} k_{2}^{i}\left(k_{2}-k^{\prime}\right)^{i^{\prime}} C\left(x_{A}, \boldsymbol{x}_{\perp}, \boldsymbol{x}_{\perp}, \boldsymbol{y}_{\perp}^{\prime}, \boldsymbol{x}_{\perp}^{\prime}\right) \\
& =\int_{\boldsymbol{x}_{\perp} \boldsymbol{x}_{\perp}^{\prime} \boldsymbol{y}_{\perp}^{\prime}} \int_{\boldsymbol{k}_{\perp}^{\prime}} e^{-i \boldsymbol{k}_{\perp}^{\prime} \boldsymbol{x}_{\perp}^{\prime}+i \boldsymbol{k}_{\perp \perp} \boldsymbol{x}_{\perp}-i\left(\boldsymbol{k}_{\perp \perp}-\boldsymbol{k}_{\perp}^{\prime}\right) \cdot \boldsymbol{y}_{\perp}^{\prime}} k_{2}^{i}\left(k_{2}-k^{\prime}\right)^{i^{\prime}} C\left(x_{A}, \boldsymbol{x}_{\perp}, \boldsymbol{x}_{\perp}, \boldsymbol{y}_{\perp}^{\prime}, \boldsymbol{x}_{\perp}^{\prime}\right) \\
& =\int_{\boldsymbol{x}_{\perp} \boldsymbol{x}_{\perp}^{\prime} \boldsymbol{y}_{\perp}^{\prime}} \int_{\boldsymbol{k}_{\perp}^{\prime}} e^{-i \boldsymbol{k}_{\perp}^{\prime} \boldsymbol{x}_{\perp}^{\prime}+i \boldsymbol{k}_{\perp \perp} \boldsymbol{x}_{\perp}-i\left(\boldsymbol{k}_{\perp \perp}-\boldsymbol{k}_{\perp}^{\prime}\right) \cdot \boldsymbol{y}_{\perp}^{\prime}} \frac{\partial^{2} C\left(x_{A}, \boldsymbol{x}_{\perp}, \boldsymbol{x}_{\perp}, \boldsymbol{y}_{\perp}^{\prime}, \boldsymbol{x}_{\perp}^{\prime}\right)}{\partial x^{i} \partial y^{\prime i^{\prime}}} \\
& =\int_{\boldsymbol{x}_{\perp} \boldsymbol{x}_{\perp}^{\prime}} e^{i \boldsymbol{k}_{2 \perp} \cdot\left(\boldsymbol{x}_{\perp}-\boldsymbol{x}_{\perp}^{\prime}\right)}\left[\frac{\partial^{2} C\left(x_{A}, \boldsymbol{x}_{\perp}, \boldsymbol{x}_{\perp}, \boldsymbol{y}_{\perp}^{\prime}, \boldsymbol{x}_{\perp}^{\prime}\right)}{\partial x^{i} \partial y^{\prime i^{\prime}}}\right]_{\boldsymbol{x}_{\perp}^{\prime}=\boldsymbol{y}_{\perp}^{\prime}} .
\end{aligned}
$$

The cross section can be written as

$$
\begin{aligned}
& \frac{d \sigma}{d^{2} \boldsymbol{p}_{\perp} d^{2} \boldsymbol{q}_{\perp} d^{2} \boldsymbol{k}_{\gamma \perp} d \eta_{p} d \eta_{q} d \eta_{k_{\gamma}}}=\frac{\alpha_{e} \alpha_{S} q_{f}^{2}}{256 \pi^{8} N_{c}\left(N_{c}^{2}-1\right)} \int_{\boldsymbol{k}_{1 \perp} \boldsymbol{k}_{2 \perp}}(2 \pi)^{2} \delta^{(2)}\left(\boldsymbol{P}_{\perp}-\boldsymbol{k}_{1 \perp}-\boldsymbol{k}_{2 \perp}\right) \frac{\varphi_{p}\left(x_{p}, k_{1 \perp}^{2}\right)}{k_{1 \perp}^{2}} \\
& \times \int_{\boldsymbol{x}_{\perp} \boldsymbol{x}_{\perp}^{\prime}} e^{i \boldsymbol{k}_{2 \perp} \cdot\left(\boldsymbol{x}_{\perp}-\boldsymbol{x}_{\perp}^{\prime}\right)} g^{\mu \mu^{\prime}} \\
& \times\left\{\operatorname{tr}\left[(\not 1+m) R_{q, \mu i}(m-\not p) \gamma^{0} R_{q, \mu^{\prime} i^{\prime}}^{\prime \dagger} \gamma^{0}\right]\left[\frac{\partial^{2} C\left(x_{A}, \boldsymbol{x}_{\perp}, \boldsymbol{y}_{\perp}, \boldsymbol{y}_{\perp}^{\prime}, \boldsymbol{x}_{\perp}^{\prime}\right)}{\partial x^{i} \partial x^{\prime i^{\prime}}}\right]_{\boldsymbol{x}_{\perp}=\boldsymbol{y}_{\perp}, \boldsymbol{x}_{\perp}^{\prime}=\boldsymbol{y}_{\perp}^{\prime}}\right. \\
& +\operatorname{tr}\left[(\not q+m) R_{q, \mu i}(m-\not p) \gamma^{0} R_{\bar{q}, \mu^{\prime} i^{\prime}}^{\dagger} \gamma^{0}\right]\left[\frac{\partial^{2} C\left(x_{A}, \boldsymbol{x}_{\perp}, \boldsymbol{y}_{\perp}, \boldsymbol{y}_{\perp}^{\prime}, \boldsymbol{x}_{\perp}^{\prime}\right)}{\partial x^{i} \partial y^{\prime i^{\prime}}}\right]_{\boldsymbol{x}_{\perp}=\boldsymbol{y}_{\perp}, \boldsymbol{x}_{\perp}^{\prime}=\boldsymbol{y}_{\perp}^{\prime}} \\
& +\operatorname{tr}\left[(\not q+m) R_{\bar{q}, \mu i}(m-\not p) \gamma^{0}{R^{\prime \prime}, \mu^{\prime} i^{\prime}}^{\prime 0} \gamma^{0}\right]\left[\frac{\partial^{2} C\left(x_{A}, \boldsymbol{x}_{\perp}, \boldsymbol{y}_{\perp}, \boldsymbol{y}_{\perp}^{\prime}, \boldsymbol{x}_{\perp}^{\prime}\right)}{\partial y^{i} \partial x^{\prime i^{\prime}}}\right]_{\boldsymbol{x}_{\perp}=\boldsymbol{y}_{\perp}, x_{\perp}^{\prime}=\boldsymbol{y}_{\perp}^{\prime}} \\
& +\operatorname{tr}\left[(\not 1+m) R_{\bar{q}, \mu i}(m-\not p) \gamma^{0} R_{\bar{q}, \mu^{\prime} i^{\prime}}^{\dagger} \gamma^{0}\right]\left[\frac{\partial^{2} C\left(x_{A}, \boldsymbol{x}_{\perp}, \boldsymbol{y}_{\perp}, \boldsymbol{y}_{\perp}^{\prime}, \boldsymbol{x}_{\perp}^{\prime}\right)}{\partial y^{i} \partial y^{\prime i^{\prime}}}\right]_{\boldsymbol{x}_{\perp}=\boldsymbol{y}_{\perp}, \boldsymbol{x}_{\perp}^{\prime}=\boldsymbol{y}_{\perp}^{\prime}} \\
& +\operatorname{tr}\left[(\not q+m) R_{q, \mu i}(m-\not p) \gamma^{0} R_{g, \mu^{\prime} i^{\prime}}^{\prime \dagger} \gamma^{0}\left[\frac{\partial^{2} C\left(x_{A}, \boldsymbol{x}_{\perp}, \boldsymbol{y}_{\perp}, \boldsymbol{x}_{\perp}^{\prime}, \boldsymbol{x}_{\perp}^{\prime}\right)}{\partial x^{i} \partial x^{\prime i^{\prime}}}\right]_{\boldsymbol{x}_{\perp}=\boldsymbol{y}_{\perp}}\right.
\end{aligned}
$$

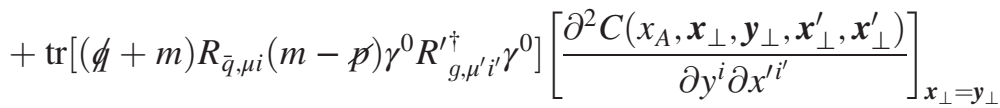

$$
\begin{aligned}
& +\operatorname{tr}\left[(\not q+m) R_{g, \mu i}(m-\not p) \gamma^{0}{R^{\prime \dagger}}_{q, \mu^{\prime} i^{\prime}}^{\prime} \gamma^{0}\right]\left[\frac{\partial^{2} C\left(x_{A}, \boldsymbol{x}_{\perp}, \boldsymbol{x}_{\perp}, \boldsymbol{y}_{\perp}^{\prime}, \boldsymbol{x}_{\perp}^{\prime}\right)}{\partial x^{i} \partial x^{\prime i^{\prime}}}\right]_{\boldsymbol{x}_{\perp}^{\prime}=\boldsymbol{y}_{\perp}^{\prime}} \\
& +\operatorname{tr}\left[(\not 1+m) R_{g, \mu i}(m-\not p) \gamma^{0}{R_{\bar{q}, \mu^{\prime} i^{\prime}}^{\prime \dagger}}^{0}\right]\left[\frac{\partial^{2} C\left(x_{A}, \boldsymbol{x}_{\perp}, \boldsymbol{x}_{\perp}, \boldsymbol{y}_{\perp}^{\prime}, \boldsymbol{x}_{\perp}^{\prime}\right)}{\partial x^{i} \partial y^{\prime i^{\prime}}}\right]_{\boldsymbol{x}_{\perp}^{\prime}=\boldsymbol{y}_{\perp}^{\prime}} \\
& \left.+\operatorname{tr}\left[(\not q+m) R_{g, \mu i}(m-\not p) \gamma^{0}{R_{g, \mu^{\prime} i^{\prime}}^{\dagger}}^{0} \gamma^{0}\right]\left[\frac{\partial^{2} C\left(x_{A}, \boldsymbol{x}_{\perp}, \boldsymbol{x}_{\perp}, \boldsymbol{x}_{\perp}^{\prime}, \boldsymbol{x}_{\perp}^{\prime}\right)}{\partial x^{i} \partial x^{\prime i^{\prime}}}\right]\right\} \text {. }
\end{aligned}
$$

The gradients of $C\left(\boldsymbol{x}_{\perp}, \boldsymbol{y}_{\perp}, \boldsymbol{y}_{\perp}^{\prime} \boldsymbol{x}_{\perp}^{\prime}\right)$ are parametrized in terms of six transverse-momentum-dependent gluon distribution functions as follows [see Eqs. (28)-(34) in [10]]:

$$
\begin{gathered}
\int_{\boldsymbol{x}_{\perp} \boldsymbol{x}_{\perp}^{\prime}} e^{i \boldsymbol{k}_{\perp \perp} \cdot\left(\boldsymbol{x}_{\perp}-\boldsymbol{x}_{\perp}^{\prime}\right)}\left[\frac{\partial^{2} C\left(x_{A}, \boldsymbol{x}_{\perp}, \boldsymbol{y}_{\perp}, \boldsymbol{y}_{\perp}^{\prime}, \boldsymbol{x}_{\perp}^{\prime}\right)}{\partial x^{i} \partial x^{\prime i^{\prime}}}\right]_{\boldsymbol{x}_{\perp}=\boldsymbol{y}_{\perp}, \boldsymbol{x}_{\perp}^{\prime}=\boldsymbol{y}_{\perp}^{\prime}} \\
=\int_{\boldsymbol{x}_{\perp} \boldsymbol{x}_{\perp}^{\prime}} e^{i \boldsymbol{k}_{\perp \perp} \cdot\left(\boldsymbol{x}_{\perp}-\boldsymbol{x}_{\perp}^{\prime}\right)}\left[\frac{\partial^{2} C\left(x_{A}, \boldsymbol{x}_{\perp}, \boldsymbol{y}_{\perp}, \boldsymbol{y}_{\perp}^{\prime}, \boldsymbol{x}_{\perp}^{\prime}\right)}{\partial y^{i} \partial y^{\prime i^{\prime}}}\right]_{x_{\perp}=\boldsymbol{y}_{\perp}, \boldsymbol{x}_{\perp}^{\prime}=\boldsymbol{y}_{\perp}^{\prime}} \\
\equiv \frac{1}{2} \delta^{i i^{\prime}} F_{1}\left(x_{A}, k_{2 \perp}^{2}\right)+\left(\frac{k_{2 \perp}^{i} k_{2 \perp}^{i^{\prime}}}{k_{2 \perp}^{2}}-\frac{1}{2} \delta^{i i^{\prime}}\right) H_{1}\left(x_{A}, k_{2 \perp}^{2}\right),
\end{gathered}
$$




$$
\begin{aligned}
& \int_{\boldsymbol{x}_{\perp} x_{\perp}^{\prime}} e^{i \boldsymbol{k}_{\perp \perp} \cdot\left(\boldsymbol{x}_{\perp}-\boldsymbol{x}_{\perp}^{\prime}\right)}\left[\frac{\partial^{2} C\left(x_{A}, \boldsymbol{x}_{\perp}, \boldsymbol{y}_{\perp}, \boldsymbol{y}_{\perp}^{\prime}, \boldsymbol{x}_{\perp}^{\prime}\right)}{\partial x^{i} \partial y^{\prime i^{\prime}}}\right]_{\boldsymbol{x}_{\perp}=\boldsymbol{y}_{\perp}, \boldsymbol{x}_{\perp}^{\prime}=\boldsymbol{y}_{\perp}^{\prime}} \\
& =\int_{\boldsymbol{x}_{\perp} \boldsymbol{x}_{\perp}^{\prime}} e^{i \boldsymbol{k}_{2 \perp} \cdot\left(\boldsymbol{x}_{\perp}-\boldsymbol{x}_{\perp}^{\prime}\right)}\left[\frac{\partial^{2} C\left(x_{A}, \boldsymbol{x}_{\perp}, \boldsymbol{y}_{\perp}, \boldsymbol{y}_{\perp}^{\prime}, \boldsymbol{x}_{\perp}^{\prime}\right)}{\partial y^{i} \partial x^{\prime i^{\prime}}}\right]_{\boldsymbol{x}_{\perp}=\boldsymbol{y}_{\perp}, \boldsymbol{x}_{\perp}^{\prime}=\boldsymbol{y}_{\perp}^{\prime}} \\
& \equiv \frac{1}{2} \delta^{i i^{\prime}} F_{2}\left(x_{A}, k_{2 \perp}^{2}\right)+\left(\frac{k_{2 \perp}^{i} k_{2 \perp}^{i^{\prime}}}{k_{2 \perp}^{2}}-\frac{1}{2} \delta^{i i^{\prime}}\right) H_{2}\left(x_{A}, k_{2 \perp}^{2}\right) \text {. } \\
& \int_{\boldsymbol{x}_{\perp} \boldsymbol{x}_{\perp}^{\prime}} e^{i \boldsymbol{k}_{\perp \perp} \cdot\left(\boldsymbol{x}_{\perp}-\boldsymbol{x}_{\perp}^{\prime}\right)}\left[\frac{\partial^{2} C\left(x_{A}, \boldsymbol{x}_{\perp}, \boldsymbol{y}_{\perp}, \boldsymbol{x}_{\perp}^{\prime}, \boldsymbol{x}_{\perp}^{\prime}\right)}{\partial x^{i} \partial x^{\prime i^{\prime}}}\right]_{\boldsymbol{x}_{\perp}=\boldsymbol{y}_{\perp}}=\int_{\boldsymbol{x}_{\perp} x_{\perp}^{\prime}} e^{i \boldsymbol{k}_{\perp \perp} \cdot\left(\boldsymbol{x}_{\perp}-\boldsymbol{x}_{\perp}^{\prime}\right)}\left[\frac{\partial^{2} C\left(x_{A}, \boldsymbol{x}_{\perp}, \boldsymbol{y}_{\perp}, \boldsymbol{x}_{\perp}^{\prime}, \boldsymbol{x}_{\perp}^{\prime}\right)}{\partial y^{i} \partial x^{\prime i^{\prime}}}\right]_{\boldsymbol{x}_{\perp}=\boldsymbol{y}_{\perp}} \\
& =\int_{\boldsymbol{x}_{\perp} x_{\perp}^{\prime}} e^{i \boldsymbol{k}_{\perp \perp} \cdot\left(\boldsymbol{x}_{\perp}-\boldsymbol{x}_{\perp}^{\prime}\right)}\left[\frac{\partial^{2} C\left(x_{A}, \boldsymbol{x}_{\perp}, \boldsymbol{x}_{\perp}, \boldsymbol{y}_{\perp}^{\prime}, \boldsymbol{x}_{\perp}^{\prime}\right)}{\partial x^{i} \partial x^{\prime i^{\prime}}}\right]_{\boldsymbol{x}_{\perp}^{\prime}=\boldsymbol{y}_{\perp}^{\prime}}=\int_{\boldsymbol{x}_{\perp} x_{\perp}^{\prime}} e^{i \boldsymbol{k}_{2 \perp} \cdot\left(\boldsymbol{x}_{\perp}-\boldsymbol{x}_{\perp}^{\prime}\right)}\left[\frac{\partial^{2} C\left(x_{A}, \boldsymbol{x}_{\perp}, \boldsymbol{x}_{\perp}, \boldsymbol{y}_{\perp}^{\prime}, \boldsymbol{x}_{\perp}^{\prime}\right)}{\partial x^{i} \partial y^{\prime i^{\prime}}}\right]_{\boldsymbol{x}_{\perp}^{\prime}=\boldsymbol{y}_{\perp}^{\prime}} \\
& =\frac{1}{2} \int_{\boldsymbol{x}_{\perp} x_{\perp}^{\prime}} e^{i k_{2 \perp} \cdot\left(\boldsymbol{x}_{\perp}-x_{\perp}^{\prime}\right)}\left[\frac{\partial^{2} C\left(x_{A}, \boldsymbol{x}_{\perp}, \boldsymbol{x}_{\perp}, \boldsymbol{x}_{\perp}^{\prime}, \boldsymbol{x}_{\perp}^{\prime}\right)}{\partial x^{i} \partial x^{\prime i^{\prime}}}\right] \equiv \frac{1}{2} \delta^{i i^{\prime}} F_{3}\left(x_{A}, k_{2 \perp}^{2}\right)+\left(\frac{k_{2 \perp}^{i} k_{2 \perp}^{i^{\prime}}}{k_{2 \perp}^{2}}-\frac{1}{2} \delta^{i i^{\prime}}\right) H_{3}\left(x_{A}, k_{2 \perp}^{2}\right) .
\end{aligned}
$$

The cross section becomes

$$
\begin{aligned}
\frac{d \sigma}{d^{2} \boldsymbol{p}_{\perp} d^{2} \boldsymbol{q}_{\perp} d^{2} \boldsymbol{k}_{\gamma \perp} d \eta_{p} d \eta_{q} d \eta_{k_{\gamma}}}= & \frac{\alpha_{e} \alpha_{S} q_{f}^{2}}{256 \pi^{8} N_{c}\left(N_{c}^{2}-1\right)} \int_{\boldsymbol{k}_{\perp \perp} \boldsymbol{k}_{2 \perp}}(2 \pi)^{2} \delta^{(2)}\left(\boldsymbol{P}_{\perp}-\boldsymbol{k}_{1 \perp}-\boldsymbol{k}_{2 \perp}\right) \frac{\varphi_{p}\left(x_{p}, k_{1 \perp}^{2}\right)}{k_{1 \perp}^{2}} \\
& \times\left\{\left(\tau_{q q, i i^{\prime}}+\tau_{\bar{q} \bar{q}, i i^{\prime}}\right)\left[\frac{1}{2} \delta^{i i^{\prime}} F_{1}\left(x_{A}, k_{2 \perp}^{2}\right)+\left(\frac{k_{2 \perp}^{i} k_{2 \perp}^{i^{\prime}}}{k_{2 \perp}^{2}}-\frac{1}{2} \delta^{i i^{\prime}}\right) H_{1}\left(x_{A}, k_{2 \perp}^{2}\right)\right]\right. \\
& +\left(\tau_{q \bar{q}, i i^{\prime}}+\tau_{\bar{q} q, i i^{\prime}}\right)\left[\frac{1}{2} \delta^{i i^{\prime}} F_{2}\left(x_{A}, k_{2 \perp}^{2}\right)+\left(\frac{k_{2 \perp}^{i} k_{2 \perp}^{i^{\prime}}}{k_{2 \perp}^{2}}-\frac{1}{2} \delta^{i i^{\prime}}\right) H_{2}\left(x_{A}, k_{2 \perp}^{2}\right)\right] \\
& +\left(\tau_{q g, i i^{\prime}}+\tau_{\bar{q} g, i i^{\prime}}+\tau_{g q, i i^{\prime}}+\tau_{g \bar{q}, i i^{\prime}}+2 \tau_{g g, i i^{\prime}}\right) \\
& \left.\times\left[\frac{1}{2} \delta^{i i^{\prime}} F_{3}\left(x_{A}, k_{2 \perp}^{2}\right)+\left(\frac{k_{2 \perp}^{i} k_{2 \perp}^{i^{\prime}}}{k_{2 \perp}^{2}}-\frac{1}{2} \delta^{i i^{\prime}}\right) H_{3}\left(x_{A}, k_{2 \perp}^{2}\right)\right]\right\}
\end{aligned}
$$

where

$$
\begin{aligned}
\tau_{a b, i i^{\prime}} & \equiv g^{\mu \mu^{\prime}} \operatorname{tr}\left[(\not q+m) R_{a, \mu i}(m-\not p) \gamma^{0} R_{b, \mu^{\prime} i^{\prime}}^{\prime \dagger} \gamma^{0}\right], \\
a, b & =q, \bar{q}, g .
\end{aligned}
$$

The $F_{i}$ and $H_{i}$ distributions, introduced in Eqs. (27)(29), have known operator definitions; see e.g. [10] where a direct relationship to the distributions in the conventional transverse-momentum-dependent (TMD) formalism discussed in [8,9] was given. Reference [10] also established a set of linear relationships to the various dipole and Weizsäcker-Williams gluon distributions [10]; see also [22]. In addition, we provide explicit expressions in terms of the BK two-point function of $A^{+}$in Appendix B.

\section{JET-PHOTON CORRELATIONS IN THE COLLINEAR APPROXIMATION}

In the following we will pick up configurations where both the photon $\boldsymbol{k}_{\gamma \perp}$ and the antiquark (we arbitrarily choose this to be the jet in this process) momentum $\boldsymbol{p}_{\perp}$ are hard, while the leftover transverse momentum of the quark is much smaller, $\boldsymbol{q}_{\perp} \ll \boldsymbol{k}_{\gamma \perp}$. The contributions where the photon is emitted within a jet can be suppressed by discarding events with a large transverse jet energy in a cone around the photon [23]. The remaining configurations are in fact dominated by collinear photon emissions from a quark line after the interaction with the nuclei. For simplicity, we will also take the collinear limit on the proton side: $\boldsymbol{k}_{1 \perp} \rightarrow 0$. The cross section becomes 


$$
\begin{aligned}
& \frac{d \sigma}{d^{2} \boldsymbol{p}_{\perp} d^{2} \boldsymbol{q}_{\perp} d^{2} \boldsymbol{k}_{\gamma \perp} d \eta_{p} d \eta_{q} d \eta_{k_{\gamma}}}=\frac{\alpha_{e} \alpha_{S} q_{f}^{2}}{512 \pi^{6} N_{c}\left(N_{c}^{2}-1\right)} x_{p} f_{g, p}\left(x_{p}, P_{\perp}^{2}\right) \\
& \times\left\{\left(\tau_{q q, i i^{\prime}}^{\mathrm{coll}}+\tau_{\bar{q} \bar{q}, i i^{\prime}}^{\mathrm{coll}}\right)\left[\frac{1}{2} \delta^{i i^{\prime}} F_{1}\left(x_{A}, P_{\perp}^{2}\right)+\left(\frac{P^{i} P^{i^{\prime}}}{P_{\perp}^{2}}-\frac{1}{2} \delta^{i i^{\prime}}\right) H_{1}\left(x_{A}, P_{\perp}^{2}\right)\right]\right. \\
& +\left(\tau_{q \bar{q}, i i^{\prime}}^{\mathrm{coll}}+\tau_{\bar{q} q, i i^{\prime}}^{\mathrm{coll}}\right)\left[\frac{1}{2} \delta^{i i^{\prime}} F_{2}\left(x_{A}, P_{\perp}^{2}\right)+\left(\frac{P^{i} P^{i^{\prime}}}{P_{\perp}^{2}}-\frac{1}{2} \delta^{i i^{\prime}}\right) H_{2}\left(x_{A}, P_{\perp}^{2}\right)\right] \\
& +\left(\tau_{q g, i i^{\prime}}^{\mathrm{coll}}+\tau_{\bar{q} g, i i^{\prime}}^{\mathrm{coll}}+\tau_{g q, i i^{\prime}}^{\mathrm{coll}}+\tau_{g \bar{q}, i i^{\prime}}^{\mathrm{coll}}+2 \tau_{g g, i i^{\prime}}^{\mathrm{coll}}\right) \\
& \left.\times\left[\frac{1}{2} \delta^{i i^{\prime}} F_{3}\left(x_{A}, P_{\perp}^{2}\right)+\left(\frac{P^{i} P^{i^{\prime}}}{P_{\perp}^{2}}-\frac{1}{2} \delta^{i i^{\prime}}\right) H_{3}\left(x_{A}, P_{\perp}^{2}\right)\right]\right\},
\end{aligned}
$$

where

$$
\begin{aligned}
& \tau_{a b, i i^{\prime}}^{\mathrm{coll}} \equiv g^{\mu \mu^{\prime}} \delta^{j j^{\prime}} \operatorname{tr}\left[(\not q+m) R_{a, \mu i j}(m-\not p) \gamma^{0} R_{b, \mu^{\prime} i^{\prime} j^{\prime} \gamma^{0}}^{\gamma^{0}}\right], \quad a, b=q, \bar{q},
\end{aligned}
$$

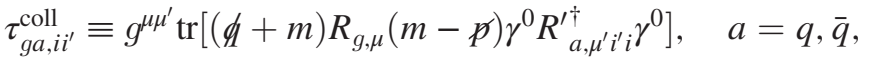

$$
\begin{aligned}
& \tau_{a g, i i^{\prime}}^{\mathrm{coll}} \equiv g^{\mu \mu^{\prime}} \operatorname{tr}\left[(\not q+m) R_{a, \mu i i^{\prime}}(m-\not p) \gamma^{0}{R^{\prime \prime}}_{g, \mu^{\prime}}^{\prime} \gamma^{0}\right], \quad a=q, \bar{q},
\end{aligned}
$$

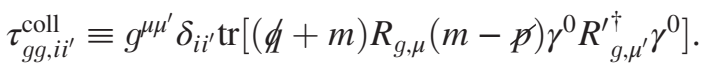

Here we have defined $R_{a}^{\mu i} \equiv k_{1}^{j} R_{a}^{\mu i j}, a=q, \bar{q}$, and $R_{g}^{\mu i} \equiv k_{1}^{i} R_{g}^{\mu}$; see Eqs. (15) and (16). Also, we introduced the collinear gluon distribution of the proton,

$$
x_{p} f_{g, p}\left(x_{p}, P_{\perp}^{2}\right) \equiv \frac{1}{4 \pi^{3}} \int_{0}^{P_{\perp}^{2}} d k_{1 \perp}^{2} \varphi_{p}\left(x_{p}, k_{1 \perp}^{2}\right) .
$$

Note that although $P_{\perp}$ is much smaller than the hard scale $\tilde{P}_{\perp}$ it is nevertheless on the order of the saturation scale of the nucleus and so the collinear approximation on the proton side should be justified [24].

The terms in the amplitude (14) containing the photon-quark collinear singularity are given by $R_{1}^{\mu i}, R_{3}^{\mu i}$ and $R_{8}^{\mu i}$; see Eq. (16). To correctly take into account this singularity the Dirac traces (33) must contain the diagonal parts corresponding to the matrices $R_{1}^{\mu i}, R_{3}^{\mu i}$ and $R_{8}^{\mu i}$, as well as interferences with the remaining part of the amplitude, leading to

$$
\begin{aligned}
& \tau_{q q, i i^{\prime}}^{\mathrm{coll}}=\tau_{33, i i^{\prime}}^{\mathrm{coll}}+\tau_{34, i i^{\prime}}^{\mathrm{coll}}+\tau_{43, i i^{\prime}}^{\mathrm{coll}}+\tau_{35, i i^{\prime}}^{\mathrm{coll}}+\tau_{53, i i^{\prime}}^{\mathrm{coll}}, \\
& \tau_{\bar{q} \bar{q}, i i^{\prime}}^{\mathrm{coll}}=\tau_{88, i i^{\prime}}^{\mathrm{coll}}+\tau_{87, i i^{\prime}}^{\mathrm{coll}}+\tau_{78, i i^{\prime}}^{\mathrm{coll}}+\tau_{86, i i^{\prime}}^{\mathrm{coll}}+\tau_{68, i i^{\prime}}^{\mathrm{coll}}, \\
& \tau_{q \bar{q}, i i^{\prime}}^{\mathrm{coll}}=\tau_{36, i i^{\prime}}^{\mathrm{coll}}+\tau_{37, i i^{\prime}}^{\mathrm{coll}}+\tau_{38, i i^{\prime}}^{\mathrm{coll}}+\tau_{48, i i^{\prime}}^{\mathrm{coll}}+\tau_{58, i i^{\prime}}^{\mathrm{coll}}, \\
& \tau_{\bar{q} q, i i^{\prime}}^{\mathrm{coll}}=\tau_{63, i i^{\prime}}^{\mathrm{coll}}+\tau_{73, i i^{\prime}}^{\mathrm{coll}}+\tau_{83, i i^{\prime}}^{\mathrm{coll}}+\tau_{84, i i^{\prime}}^{\mathrm{coll}}+\tau_{85, i i^{\prime}}^{\mathrm{coll}}, \\
& \tau_{g q, i i^{\prime}}^{\mathrm{coll}}=\tau_{13, i i^{\prime}}^{\mathrm{coll}}+\tau_{14, i i^{\prime}}^{\mathrm{coll}}+\tau_{15, i i^{\prime}}^{\mathrm{coll}}+\tau_{23, i i^{\prime}}^{\mathrm{coll}}, \\
& \tau_{g \bar{q}, i i^{\prime}}^{\mathrm{coll}}=\tau_{16, i i^{\prime}}^{\mathrm{coll}}+\tau_{17, i i^{\prime}}^{\mathrm{coll}}+\tau_{18, i i^{\prime}}^{\mathrm{coll}}+\tau_{28, i i^{\prime}}^{\mathrm{coll}}, \\
& \tau_{q g, i i^{\prime}}^{\mathrm{coll}}=\tau_{31, i i^{\prime}}^{\mathrm{coll}}+\tau_{41, i i^{\prime}}^{\mathrm{coll}}+\tau_{51, i i^{\prime}}^{\mathrm{coll}}+\tau_{32, i i^{\prime}}^{\mathrm{coll}}, \\
& \tau_{\bar{q} g, i i^{\prime}}^{\mathrm{coll}}=\tau_{61, i i^{\prime}}^{\mathrm{coll}}+\tau_{71, i i^{\prime}}^{\mathrm{coll}}+\tau_{81, i i^{\prime}}^{\mathrm{coll}}+\tau_{82, i i^{\prime}}^{\mathrm{coll}}, \\
& \tau_{g g, i i^{\prime}}^{\text {coll }}=\tau_{11, i i^{\prime}}^{\text {coll }}+\tau_{12, i i^{\prime}}^{\text {coll }}+\tau_{21, i i^{\prime}}^{\text {coll }} \text {. }
\end{aligned}
$$

Here we have defined

$$
\begin{aligned}
& \tau_{\alpha \beta, i i^{\prime}}^{\mathrm{coll}} \equiv g^{\mu \mu^{\prime}} \delta^{j j^{\prime}} \operatorname{tr}\left[(\not q+m) R_{\alpha, \mu i j}(m-\not p) \gamma^{0} R_{\beta, \mu^{\prime} i^{\prime} j^{\prime}}^{\prime \gamma^{0}}\right], \quad \alpha, \beta=3, \ldots, 8, \\
& \tau_{\alpha \beta, i i^{\prime}}^{\mathrm{coll}} \equiv g^{\mu \mu^{\prime}} \operatorname{tr}\left[(\not 1+m) R_{\alpha, \mu}(m-\not p) \gamma^{0}{R^{\prime}}_{\beta, \mu^{\prime} i^{\prime} i}^{\dagger} \gamma^{0}\right], \quad \alpha=1,2, \beta=3, \ldots, 8, \\
& \tau_{\alpha \beta, i i^{\prime}}^{\mathrm{coll}} \equiv g^{\mu \mu^{\prime}} \operatorname{tr}\left[(\not q+m) R_{\alpha, \mu i i^{\prime}}(m-\not p) \gamma^{0} R_{\beta, \mu^{\prime}}^{\prime \dagger} \gamma^{0}\right], \quad \alpha=3, \ldots, 8, \beta=1,2 \text {, } \\
& \tau_{\alpha \beta, i i^{\prime}}^{\mathrm{coll}} \equiv g^{\mu \mu^{\prime}} \delta_{i i^{\prime}} \operatorname{tr}\left[(\not 1+m) R_{\alpha, \mu}(m-\not p) \gamma^{0} R_{\beta, \mu^{\prime}}^{\prime \dagger} \gamma^{0}\right], \quad \alpha, \beta=1,2,
\end{aligned}
$$


where $R_{\alpha}^{\mu i} \equiv k_{1}^{j} R_{\alpha}^{\mu i j}, \alpha=3, \ldots, 8$, and $R_{\alpha}^{\mu i} \equiv k_{1}^{i} R_{\alpha}^{\mu}, \alpha=1$, 2; see Eq. (15).

\section{A. Integration over the photon-quark collinear singularity}

We introduce the photon momentum fraction

$$
z \equiv \frac{k_{\gamma}^{+}}{k_{\gamma}^{+}+q^{+}}
$$

and separate out the collinear singularity in the Dirac traces:

$$
\tau_{a b, i i^{\prime}}^{\mathrm{coll}}\left(\boldsymbol{w}_{\perp}\right) \equiv \frac{\tau_{a b, i i^{\prime}}^{\mathrm{coll}, \mathrm{reg}}\left(\boldsymbol{w}_{\perp}\right)}{\left[z \boldsymbol{w}_{\perp}-\boldsymbol{k}_{\gamma \perp}\right]^{2}}
$$

The integral over the transverse phase space $\boldsymbol{q}_{\perp}$ is dominated by the singularity $\boldsymbol{w}_{\perp} \equiv \boldsymbol{q}_{\perp}+\boldsymbol{k}_{\gamma \perp}=\boldsymbol{k}_{\gamma \perp} / z$. The integral is calculated by expanding the integrand around this singularity and then integrating over it.

A generic integral is

$$
\begin{aligned}
I & =\int_{\boldsymbol{q}_{\perp}} \frac{F\left(\boldsymbol{w}_{\perp}, \boldsymbol{p}_{\perp}\right)}{\left(z \boldsymbol{w}_{\perp}-\boldsymbol{k}_{\gamma \perp}\right)^{2}\left(\boldsymbol{w}_{\perp}+\boldsymbol{p}_{\perp}\right)^{2}} \\
& =\int_{\boldsymbol{w}_{\perp}} \frac{F\left(\boldsymbol{w}_{\perp}, \boldsymbol{p}_{\perp}\right)}{\left(z \boldsymbol{w}_{\perp}-\boldsymbol{k}_{\gamma \perp}\right)^{2}\left(\boldsymbol{w}_{\perp}+\boldsymbol{p}_{\perp}\right)^{2}},
\end{aligned}
$$

where in the second line we shifted the integration $\boldsymbol{q}_{\perp} \rightarrow \boldsymbol{w}_{\perp} . F\left(\boldsymbol{w}_{\perp}, \boldsymbol{p}_{\perp}\right)$ is a function containing the hard parts that potentially depend on $\boldsymbol{w}_{\perp}$ and $\boldsymbol{p}_{\perp}$ as well as the distribution function that depends on $\boldsymbol{P}_{\perp}=\boldsymbol{w}_{\perp}+\boldsymbol{p}_{\perp}$. In (39) we have separated out explicitly the perturbative tail $\sim 1 / P_{\perp}^{2}$ of the distribution functions. Expanding $F\left(\boldsymbol{w}_{\perp}, \boldsymbol{p}_{\perp}\right)$ around the collinear singularity we obtain

$$
I \simeq F\left(\boldsymbol{k}_{\gamma \perp} / z, \boldsymbol{p}_{\perp}\right) \int_{\boldsymbol{w}_{\perp}} \frac{1}{\left(z \boldsymbol{w}_{\perp}-\boldsymbol{k}_{\gamma \perp}\right)^{2}\left(\boldsymbol{w}_{\perp}+\boldsymbol{p}_{\perp}\right)^{2}}=\frac{1}{2 \pi} \frac{F\left(\boldsymbol{k}_{\gamma \perp} / z, \boldsymbol{p}_{\perp}\right)}{\left(\boldsymbol{k}_{\gamma \perp}+z \boldsymbol{p}_{\perp}\right)^{2}}\left\{-\frac{1}{\epsilon}+\gamma_{E}+\log \left[\frac{\left(\boldsymbol{k}_{\gamma \perp}+z \boldsymbol{p}_{\perp}\right)^{2}}{4 \pi z^{2} \mu^{2}}\right]+O(\epsilon)\right\},
$$

where in the second line we evaluated the integral in $d$ dimensions and expanded around $d=2-2 \epsilon$. Here $\mu^{2}$ is the renormalization scale. Subtracting the $1 / \epsilon$ divergence and defining $\mu^{2}=\mu_{\overline{\mathrm{MS}}}^{2} e^{\gamma_{E}} / 4 \pi$, we obtain the final formula for the integral in the $\overline{\mathrm{MS}}$ scheme:

$$
I_{\overline{\mathrm{MS}}}=\frac{1}{2 \pi} \frac{F\left(\boldsymbol{k}_{\gamma \perp} / z, \boldsymbol{p}_{\perp}\right)}{\left(\boldsymbol{k}_{\gamma \perp}+z \boldsymbol{p}_{\perp}\right)^{2}} \log \left[\frac{\left(\boldsymbol{k}_{\gamma \perp}+z \boldsymbol{p}_{\perp}\right)^{2}}{z^{2} \mu_{\overline{\mathrm{MS}}}^{2}}\right] .
$$

Using (41) we calculate the $\boldsymbol{q}_{\perp}$ integrals in the cross section as

$$
\begin{aligned}
\int_{\boldsymbol{q}_{\perp}} & \frac{\tau_{a b, i i^{\prime}}^{\mathrm{coll}}\left(\boldsymbol{w}_{\perp}\right)}{\left(\boldsymbol{w}_{\perp}+\boldsymbol{p}_{\perp}\right)^{2}} P_{\perp}^{2}\left[\frac{1}{2} \delta^{i i^{\prime}} F_{j}\left(x_{A}, P_{\perp}^{2}\right)+\left(\frac{P^{i} P^{i^{\prime}}}{P_{\perp}^{2}}-\frac{1}{2} \delta^{i i^{\prime}}\right) H_{j}\left(x_{A}, P_{\perp}^{2}\right)\right] \\
= & \frac{1}{2 \pi} \frac{1}{z^{2}} \frac{1}{\left(\boldsymbol{p}_{\perp}+\boldsymbol{k}_{\gamma \perp} / z\right)^{2}} \log \left[\frac{\left(\boldsymbol{p}_{\perp}+\boldsymbol{k}_{\gamma \perp} / z\right)^{2}}{\mu_{\overline{\mathrm{MS}}}^{2}}\right] \\
& \times \tau_{a b, i i^{\prime}}^{\mathrm{coll}, \mathrm{reg}}\left(\boldsymbol{k}_{\gamma \perp} / z\right)\left(\boldsymbol{p}_{\perp}+\boldsymbol{k}_{\gamma \perp} / z\right)^{2}\left[\frac{1}{2} \delta^{i i^{\prime}} F_{j}\left(x_{A},\left(\boldsymbol{p}_{\perp}+\boldsymbol{k}_{\gamma \perp} / z\right)^{2}\right)\right. \\
& \left.+\left(\frac{\left(p^{i}+k_{\gamma}^{i} / z\right)\left(p^{i^{\prime}}+k_{\gamma}^{i^{\prime}} / z\right)}{\left(\boldsymbol{p}_{\perp}+\boldsymbol{k}_{\gamma \perp} / z\right)^{2}}-\frac{1}{2} \delta^{i i^{\prime}}\right) H_{j}\left(x_{A},\left(\boldsymbol{p}_{\perp}+\boldsymbol{k}_{\gamma \perp} / z\right)^{2}\right)\right] .
\end{aligned}
$$

\section{B. Cross section in the correlation limit}

We calculate $\tau_{a b, i i^{\prime}}^{\text {coll, reg }}\left(\boldsymbol{k}_{\gamma \perp} / z\right)$ assuming that the total momentum of the final state in the correlation limit, $\boldsymbol{p}_{\perp}+\boldsymbol{k}_{\gamma \perp} / z$, is small. This follows from the fact that the hard factors have been expanded around $\boldsymbol{k}_{1 \perp}, \boldsymbol{k}_{2 \perp} \rightarrow 0$ and from momentum conservation. We introduce the new variables

$$
\boldsymbol{Q}_{\perp} \equiv \boldsymbol{k}_{\gamma \perp}+\boldsymbol{p}_{\perp}, \quad \tilde{\boldsymbol{P}}_{\perp} \equiv \frac{1}{2}\left(\boldsymbol{p}_{\perp}-\boldsymbol{k}_{\gamma \perp}\right) .
$$

Below we will consider the limit where $Q_{\perp} \ll \tilde{P}_{\perp}$. Since this means that both $Q_{\perp}$ and $\left|\boldsymbol{p}_{\perp}+\boldsymbol{k}_{\gamma \perp} / z\right|$ are small, we are considering events where the photon picks up most of the energy of its parent quark, so that $z$ is close to unity. 
The explicit expressions for the hard factors are

$$
\begin{aligned}
& \tau_{q q, i i^{\prime}}^{\mathrm{coll}, \mathrm{reg}}\left(\boldsymbol{k}_{\gamma \perp} / z\right)+\tau_{\bar{q} \bar{q}, i i^{\prime}}^{\mathrm{coll}, \mathrm{reg}}\left(\boldsymbol{k}_{\gamma \perp} / z\right)=\frac{2 \zeta z^{2}(1-z)(1+z)^{4}}{(\zeta+z)^{4}} \frac{1}{\tilde{P}_{\perp}^{4}}\left(\left(\zeta^{2}+z^{2}\right) \delta_{i i^{\prime}}-\frac{4 \zeta z(\zeta-z)^{2}}{(\zeta+z)^{2}} \frac{\tilde{P}_{i} \tilde{P}_{i^{\prime}}}{\tilde{P}_{\perp}^{2}}\right) \frac{1+(1-z)^{2}}{z}, \\
& \tau_{q \bar{q}, i i^{\prime}}^{\mathrm{coll}, \mathrm{reg}}\left(\boldsymbol{k}_{\gamma \perp} / z\right)+\tau_{\bar{q} q, i i^{\prime}}^{\mathrm{coll}, \mathrm{reg}}\left(\boldsymbol{k}_{\gamma \perp} / z\right)=-\frac{8 \zeta^{2} z^{3}(1-z)(1+z)^{4}(\zeta-z)^{2}}{(\zeta+z)^{6}} \frac{\tilde{P}_{i} \tilde{P}_{i^{\prime}}}{\tilde{P}_{\perp}^{6}} \frac{1+(1-z)^{2}}{z}, \\
& \tau_{g q, i i^{\prime}}^{\mathrm{coll}, \mathrm{reg}}\left(\boldsymbol{k}_{\gamma \perp} / z\right)+\tau_{q g, i i^{\prime}}^{\mathrm{coll}, \mathrm{reg}}\left(\boldsymbol{k}_{\gamma \perp} / z\right)+\tau_{g \bar{q}, i i^{\prime}}^{\mathrm{coll,} \text { reg }}\left(\boldsymbol{k}_{\gamma \perp} / z\right)+\tau_{\bar{q} g, i i^{\prime}}^{\mathrm{coll}, \mathrm{reg}}\left(\boldsymbol{k}_{\gamma \perp} / z\right)+2 \tau_{g g, i i^{\prime}}^{\mathrm{coll}, \mathrm{reg}}\left(\boldsymbol{k}_{\gamma \perp} / z\right) \\
& =-\frac{4 \zeta^{2} z^{3}(1-z)(1+z)^{4}}{(\zeta+z)^{6}} \frac{1}{\tilde{P}_{\perp}^{4}}\left(\left(\zeta^{2}+z^{2}\right) \delta_{i i^{\prime}}-2(\zeta-z)^{2} \frac{\tilde{P}_{i} \tilde{P}_{i^{\prime}}}{\tilde{P}_{\perp}^{2}}\right) \frac{1+(1-z)^{2}}{z},
\end{aligned}
$$

where we see explicitly the photon splitting function $\left(1+(1-z)^{2}\right) / z$. We also introduced the abbreviation

$$
\zeta \equiv \frac{k_{\gamma}^{+}}{p^{+}}=\frac{k_{\gamma \perp}}{p_{\perp}} e^{\eta_{k_{\gamma}}-\eta_{p}} .
$$

In terms of $\boldsymbol{Q}_{\perp}$ and $\tilde{\boldsymbol{P}}_{\perp}$ the transverse momentum in the nuclear gluon distributions is

$$
\boldsymbol{p}_{\perp}+\frac{1}{z} \boldsymbol{k}_{\gamma \perp}=-\frac{1-z}{z} \tilde{\boldsymbol{P}}_{\perp}+\frac{1+z}{2 z} \boldsymbol{Q}_{\perp} .
$$

Next, we multiply the transverse projector in Eq. (42) with $\tilde{P}_{i} \tilde{P}_{i^{\prime}}$ from (44):

$$
\begin{aligned}
\left(\frac{\left(p^{i}+k_{\gamma}^{i} / z\right)\left(p^{i^{\prime}}+k_{\gamma}^{i^{\prime}} / z\right)}{\left(\boldsymbol{p}_{\perp}+\boldsymbol{k}_{\gamma \perp} / z\right)^{2}}-\frac{1}{2} \delta^{i i^{\prime}}\right) \frac{\tilde{P}_{i} \tilde{P}_{i^{\prime}}}{\tilde{P}_{\perp}^{2}} & =\frac{\left(-\frac{1-z}{z} \tilde{P}_{\perp}^{2}+\frac{1+z}{2 z} \boldsymbol{Q}_{\perp} \cdot \tilde{\boldsymbol{P}}_{\perp}\right)^{2}}{\left(-\frac{1-z}{z} \tilde{\boldsymbol{P}}_{\perp}+\frac{1+z}{2 z} \boldsymbol{Q}_{\perp}\right)^{2} \tilde{P}_{\perp}^{2}}-\frac{1}{2} \\
& \simeq \frac{1}{2}-\frac{(1+z)^{2}}{4(1-z)^{2}} \frac{Q_{\perp}^{2}}{\tilde{P}_{\perp}^{2}}+\frac{(1+z)^{2}}{4(1-z)^{2}} \frac{\left(\boldsymbol{Q}_{\perp} \cdot \tilde{\boldsymbol{P}}_{\perp}\right)^{2}}{\tilde{P}_{\perp}^{4}}
\end{aligned}
$$

We took the correlation limit $Q_{\perp} \ll \tilde{P}_{\perp}$ in the last line but in the cross section below we will keep the full expression. The full cross section, integrated over the quark momentum $\boldsymbol{q}_{\perp}$, is

$$
\begin{aligned}
\frac{d \sigma}{d^{2} \tilde{\boldsymbol{P}}_{\perp} d^{2} \boldsymbol{Q}_{\perp} d \eta_{p} d \eta_{k_{\gamma}} d z}= & \frac{\alpha_{e} \alpha_{S} q_{f}^{2}}{64 \pi^{4} N_{c}\left(N_{c}^{2}-1\right)} x_{p} f_{g, p}\left(x_{p},\left(-\frac{1-z}{z} \tilde{\boldsymbol{P}}_{\perp}+\frac{1+z}{2 z} \boldsymbol{Q}_{\perp}\right)^{2}\right) \\
& \times \frac{1}{2 \pi} \frac{1+(1-z)^{2}}{z} \log \left[\frac{\left(-\frac{1-z}{z} \tilde{\boldsymbol{P}}_{\perp}+\frac{1+z}{2 z} \boldsymbol{Q}_{\perp}\right)^{2}}{\Lambda_{\overline{\mathrm{MS}}}^{2}}\right] \\
& \times \frac{\zeta(1+z)^{4}}{z(\zeta+z)^{6}} \frac{1}{\tilde{P}_{\perp}^{4}}\left\{\left(\zeta^{4}+6 \zeta^{2} z^{2}+z^{4}\right) F_{1}\left(x_{A},\left(-\frac{1-z}{z} \tilde{\boldsymbol{P}}_{\perp}+\frac{1+z}{2 z} \boldsymbol{Q}_{\perp}\right)^{2}\right)\right. \\
& -2 \zeta z(\zeta-z)^{2} F_{2}\left(x_{A},\left(-\frac{1-z}{z} \tilde{\boldsymbol{P}}_{\perp}+\frac{1+z}{2 z} \boldsymbol{Q}_{\perp}\right)^{2}\right)-4 \zeta^{2} z^{2} F_{3}\left(x_{A},\left(-\frac{1-z}{z} \tilde{\boldsymbol{P}}_{\perp}+\frac{1+z}{2 z} \boldsymbol{Q}_{\perp}\right)^{2}\right) \\
& +4 \zeta z(\zeta-z)^{2}\left[\frac{\frac{(1-z)^{2}}{z^{2}} \tilde{\boldsymbol{P}}_{\perp}^{2}+\frac{(1+z)^{2}}{4 z^{2}} \frac{\left(\boldsymbol{Q}_{\perp} \tilde{\boldsymbol{P}}_{\perp}\right)^{2}}{\left(\tilde{\boldsymbol{P}}_{\perp}^{2}\right.}-\frac{1-z^{2}}{z^{2}} \boldsymbol{Q}_{\perp} \cdot \tilde{\boldsymbol{P}}_{\perp}}{z}-\frac{1}{2}\right] \\
& \times\left[-H_{1}\left(x_{A},\left(-\frac{1+z}{2 z} \boldsymbol{Q}_{\perp}\right)^{2}\right.\right. \\
& \left.\left.+H_{3}\left(x_{A},\left(-\frac{1-z}{z} \tilde{\boldsymbol{P}}_{\perp}+\frac{1+z}{2 z} \boldsymbol{Q}_{\perp}\right)^{2}\right)-H_{2}\left(x_{A},\left(-\frac{1+z}{2 z} \boldsymbol{Q}_{\perp}\right)^{2}\right)\right]\right\}
\end{aligned}
$$

Here we have added the contribution to the cross section coming from the fragmentation photons proportional to the quarkphoton fragmentation function 


$$
D_{q \rightarrow \gamma}\left(z, \mu^{2}\right)=\frac{1}{2 \pi} \frac{1+(1-z)^{2}}{z} \log \left(\frac{\mu^{2}}{\Lambda_{\overline{\mathrm{MS}}}^{2}}\right)
$$

That way, the cross section is independent of the arbitrary renormalization scale $\mu^{2}$.

It follows from (B6) that the combination of linearly polarized gluon distributions that appears in the cross section vanishes:

$$
-H_{1}\left(x_{A}, k_{\perp}^{2}\right)-H_{2}\left(x_{A}, k_{\perp}^{2}\right)+H_{3}\left(x_{A}, k_{\perp}^{2}\right)=0
$$

We now expand the cross section in powers of ${Q_{\perp}}^{2}$ :

$$
\begin{aligned}
\frac{d \sigma}{d^{2} \tilde{\boldsymbol{P}}_{\perp} d^{2} \boldsymbol{Q}_{\perp} d \eta_{p} d \eta_{k_{\gamma}} d z}= & \frac{\alpha_{e} \alpha_{S} q_{f}^{2}}{64 \pi^{4} N_{c}\left(N_{c}^{2}-1\right)} \frac{1}{z(1-z)} x_{p} f_{g, p}\left(x_{p}, \frac{(1-z)^{2}}{z^{2}} \tilde{P}_{\perp}^{2}\right) \frac{1}{2 \pi} \frac{1+(1-z)^{2}}{z} \log \left[\frac{(1-z)^{2} \tilde{P}_{\perp}^{2}}{z^{2} \Lambda_{\overline{\mathrm{MS}}}^{2}}\right] \\
& \times \frac{\zeta(1-z)(1+z)^{4}}{(\zeta+z)^{6}} \frac{1}{\tilde{P}_{\perp}^{4}} \sum_{n=0}^{\infty} \frac{1}{n !}\left\{\left(\zeta^{4}+6 \zeta^{2} z^{2}+z^{4}\right)\left[\frac{\partial^{n} F_{1}}{\partial Q^{i_{1}} \ldots \partial Q^{i_{n}}}\right]_{Q_{\perp}=0}\right. \\
& \left.-2 \zeta z(\zeta-z)^{2}\left[\frac{\partial^{n} F_{2}}{\partial Q^{i_{1}} \ldots \partial Q^{i_{n}}}\right]_{Q_{\perp}=0}-4 \zeta^{2} z^{2}\left[\frac{\partial^{n} F_{3}}{\partial Q^{i_{1}} \ldots \partial Q^{i_{n}}}\right]_{Q_{\perp}=0}\right\} Q^{i_{1}} \ldots Q^{i_{n}} .
\end{aligned}
$$

In this expansion we have neglected the dependence of $x_{p}$ and $x_{A}$ on $Q_{\perp}$ in the proton and the nuclear distributions. In both cases the derivatives will bring energy denominators that suppress such contributions in the high-energy limit. In addition, the derivative of the proton distribution over $\log 1 / x_{p}$ would be $\alpha_{S}$ suppressed by virtue of the Balitsky-Fadin-KuraevLipatov (BFKL) equation [25]. The nuclear distributions evolve according to the BK equation which leads to an even stronger suppression for transverse momenta of order (or less than) the saturation scale of the nucleus.

Evaluating the derivatives of any of the $F_{j}$ we get

$$
\left[\frac{\partial F_{j}}{\partial Q^{i_{1}} \ldots \partial Q^{i_{n}}}\right]_{Q_{\perp}=0} Q^{i_{1}} \ldots Q^{i_{n}}=(-i)^{n} \frac{(1+z)^{n}}{(2 z)^{n}}\left(\pi R_{A}^{2}\right) \int_{\boldsymbol{x}_{\perp}}\left(\boldsymbol{Q}_{\perp} \cdot \boldsymbol{x}_{\perp}\right)^{n} \exp \left(i \frac{1-z}{z} \tilde{\boldsymbol{P}}_{\perp} \cdot \boldsymbol{x}_{\perp}\right) \tilde{F}_{j}\left(x_{A}, x_{\perp}^{2}\right),
$$

where $\tilde{F}_{j}\left(x_{A}, x_{\perp}^{2}\right)$ is the Fourier transform of the distribution functions $F_{j}\left(x_{A}, k_{\perp}^{2}\right)$. For $n=1,2$ we find

$$
\left[\frac{\partial F_{j}}{\partial Q^{i_{1}}}\right]_{Q_{\perp}=0} Q^{i_{1}}=\cos \phi \frac{1+z}{2 z} \frac{z}{1-z} \frac{Q_{\perp}}{\tilde{P}_{\perp}} F_{j}^{(1,1)}\left(x_{A}, \frac{(1-z)^{2}}{z^{2}} \tilde{P}_{\perp}^{2}\right)
$$

and

$$
\left[\frac{\partial^{2} F_{j}}{\partial Q^{i_{1}} \partial Q^{i_{2}}}\right]_{Q_{\perp}=0} Q^{i_{1}} Q^{i_{2}}=\frac{1}{2} \frac{(1+z)^{2}}{4 z^{2}} \frac{z^{2}}{(1-z)^{2}} \frac{Q_{\perp}^{2}}{\tilde{P}_{\perp}^{2}}\left[-F_{j}^{(0,2)}\left(x_{A}, \frac{(1-z)^{2}}{z^{2}} \tilde{P}_{\perp}^{2}\right)+\cos 2 \phi F_{j}^{(2,2)}\left(x_{A}, \frac{(1-z)^{2}}{z^{2}} \tilde{P}_{\perp}^{2}\right)\right],
$$

where

$$
F_{j}^{(a, b)}\left(x_{A}, k_{\perp}^{2}\right) \equiv\left(\pi R_{A}^{2}\right) 2 \pi \int_{0}^{\infty} x_{\perp} d x_{\perp} J_{a}\left(k_{\perp} x_{\perp}\right)\left(x_{\perp} k_{\perp}\right)^{b} \tilde{F}_{j}\left(x_{\perp}^{2}\right)
$$

and where $\cos \phi \equiv \boldsymbol{Q}_{\perp} \cdot \tilde{\boldsymbol{P}}_{\perp} /\left(Q_{\perp} \tilde{P}_{\perp}\right)$. Note that $F_{j}^{(0,0)}\left(x_{A}, k_{\perp}^{2}\right)=F_{j}\left(x_{A}, k_{\perp}^{2}\right)$. The cross section, expanded up to the order $Q_{\perp}^{2} / P_{\perp}^{2}$, is

\footnotetext{
${ }^{2}$ In fact, the expansion is in powers of $\frac{1+z}{1-z} Q_{\perp} / \tilde{P}_{\perp}$. Hence, for large $z$ the expansions given below apply only for rather small values of $Q_{\perp} / \tilde{P}_{\perp}$. For larger $Q_{\perp} / \tilde{P}_{\perp}$ one could compute the angular correlations numerically from Eq. (48).
} 


$$
\begin{aligned}
\frac{d \sigma}{d^{2} \tilde{\boldsymbol{P}}_{\perp} d^{2} \boldsymbol{Q}_{\perp} d \eta_{p} d \eta_{k_{\gamma}} d z}= & \frac{\alpha_{e} \alpha_{S} q_{f}^{2}}{64 \pi^{4} N_{c}\left(N_{c}^{2}-1\right)} x_{p} f_{g, p}\left(x_{p}, \frac{(1-z)^{2}}{z^{2}} \tilde{P}_{\perp}^{2}\right) \frac{1}{2 \pi} \frac{1+(1-z)^{2}}{z} \log \left[\frac{(1-z)^{2} \tilde{P}_{\perp}^{2}}{z^{2} \Lambda_{\overline{\mathrm{MS}}}^{2}}\right] \\
& \times \frac{\zeta(1+z)^{4}}{z(\zeta+z)^{6}} \frac{1}{\tilde{P}_{\perp}^{4}}\left\{( \zeta ^ { 4 } + 6 \zeta ^ { 2 } z ^ { 2 } + z ^ { 4 } ) \left[F_{1}\left(x_{A}, \frac{(1-z)^{2}}{z^{2}} P_{\perp}^{2}\right)-\frac{1}{4} \frac{(1+z)^{2}}{4 z^{2}} \frac{z^{2}}{(1-z)^{2}} \frac{Q_{\perp}^{2}}{\tilde{P}_{\perp}^{2}} F_{1}^{(0,2)}\right.\right. \\
& \left.\times\left(x_{A}, \frac{(1-z)^{2}}{z^{2}} P_{\perp}^{2}\right)\right] \\
& -2 \zeta z(\zeta-z)^{2}\left[F_{2}\left(x_{A}, \frac{(1-z)^{2}}{z^{2}} P_{\perp}^{2}\right)-\frac{1}{4} \frac{(1+z)^{2}}{4 z^{2}} \frac{z^{2}}{(1-z)^{2}} \frac{Q_{\perp}^{2}}{\tilde{P}_{\perp}^{2}} F_{2}^{(0,2)}\left(x_{A}, \frac{(1-z)^{2}}{z^{2}} P_{\perp}^{2}\right)\right] \\
& -4 \zeta^{2} z^{2}\left[F_{3}\left(x_{A}, \frac{(1-z)^{2}}{z^{2}} P_{\perp}^{2}\right)-\frac{1}{4} \frac{(1+z)^{2}}{4 z^{2}} \frac{z^{2}}{(1-z)^{2}} \frac{Q_{\perp}^{2}}{\tilde{P}_{\perp}^{2}} F_{3}^{(0,2)}\left(x_{A}, \frac{(1-z)^{2}}{z^{2}} P_{\perp}^{2}\right)\right] \\
& +\cos \phi \frac{1+z}{2 z} \frac{z}{1-z} \frac{Q_{\perp}}{\tilde{P}}\left[\left(\zeta_{\perp}^{4}+6 \zeta^{2} z^{2}+z^{4}\right) F_{1}^{(1,1)}\left(x_{A}, \frac{(1-z)^{2}}{z^{2}} P_{\perp}^{2}\right)-2 \zeta z(\zeta-z)^{2} F_{2}^{(1,1)}\right. \\
& \left.\times\left(x_{A}, \frac{(1-z)^{2}}{z^{2}} P_{\perp}^{2}\right)-4 \zeta^{2} z^{2} F_{3}^{(1,1)}\left(x_{A}, \frac{(1-z)^{2}}{z^{2}} P_{\perp}^{2}\right)\right] \\
& +\frac{1}{4} \cos 2 \phi \frac{(1+z)^{2}}{4 z^{2}} \frac{z^{2}}{(1-z)^{2}} \frac{Q_{\perp}^{2}}{\tilde{P}_{\perp}^{2}}\left[\left(\zeta^{4}+6 \zeta^{2} z^{2}+z^{4}\right) F_{1}^{(2,2)}\left(x_{A}, \frac{(1-z)^{2}}{z^{2}} P_{\perp}^{2}\right)-2 \zeta z(\zeta-z)^{2} F_{2}^{(2,2)}\right. \\
& \left.\left.\times\left(x_{A}, \frac{(1-z)^{2}}{z^{2}} P_{\perp}^{2}\right)-4 \zeta^{2} z^{2} F_{3}^{(2,2)}\left(x_{A}, \frac{(1-z)^{2}}{z^{2}} P_{\perp}^{2}\right)\right]+O\left(Q_{\perp}^{3} / \tilde{P}_{\perp}^{3}\right)\right\}
\end{aligned}
$$

In the collinear approximation $x_{p, A}$ become

$$
\begin{gathered}
x_{p} \sqrt{s}=p_{\perp} e^{\eta_{p}}+z k_{\gamma \perp} e^{\eta_{k_{\gamma}}}, \\
x_{A} \sqrt{s}=p_{\perp} e^{-\eta_{p}}+\frac{1}{z} k_{\gamma \perp} e^{-\eta_{k_{\gamma}}} .
\end{gathered}
$$

\section{Angular correlations}

We can define angular correlations via

$$
a_{n} \equiv\langle\cos (n \phi)\rangle \equiv \frac{\int_{0}^{2 \pi} d \phi \frac{d \sigma}{d^{2} \tilde{\boldsymbol{P}}_{\perp} d^{2} \boldsymbol{Q}_{\perp} d \eta_{p} d \eta_{k_{\gamma}} d z} \cos (n \phi)}{\int_{0}^{2 \pi} d \phi \frac{d \sigma}{d^{2} \tilde{\boldsymbol{P}}_{\perp} d^{2} \boldsymbol{Q}_{\perp} d \eta_{p} d \eta_{k_{\gamma}} d z}},
$$

where $\phi$ is the angle between the photon and jet transverse momentum imbalance $\boldsymbol{Q}_{\perp}$ and their average transverse momentum $\tilde{\boldsymbol{P}}_{\perp}$. In principle, all $a_{n}$ can be nonzero but are increasingly suppressed like $\left(Q_{\perp} / \tilde{P}_{\perp}\right)^{n}$. From (56) we can obtain $a_{1}$ and $a_{2}$ as follows:

$$
\begin{gathered}
a_{1}=\frac{1+z}{4(1-z)} \frac{\left.Q_{\perp}\right)}{\tilde{P}_{\perp}} \frac{\left(\zeta^{4}+6 \zeta^{2} z^{2}+z^{4}\right) F_{1}^{(1,1)}-2 \zeta z(\zeta-z)^{2} F_{2}^{(1,1)}-4 \zeta^{2} z^{2} F_{3}^{(1,1)}}{\left(\zeta^{4}+6 \zeta^{2} z^{2}+z^{4}\right) F_{1}-2 \zeta z(\zeta-z)^{2} F_{2}-4 \zeta^{2} z^{2} F_{3}}+O\left(Q_{\perp}^{3} / \tilde{P}_{\perp}^{3}\right), \\
a_{2}=\frac{(1+z)^{2}}{32(1-z)^{2}} \frac{Q_{\perp}^{2}}{\tilde{P}_{\perp}^{2}} \frac{\left(\zeta^{4}+6 \zeta^{2} z^{2}+z^{4}\right) F_{1}^{(2,2)}-2 \zeta z(\zeta-z)^{2} F_{2}^{(2,2)}-4 \zeta^{2} z^{2} F_{3}^{(2,2)}}{\left(\zeta^{4}+6 \zeta^{2} z^{2}+z^{4}\right) F_{1}-2 \zeta z(\zeta-z)^{2} F_{2}-4 \zeta^{2} z^{2} F_{3}}+O\left(Q_{\perp}^{3} / \tilde{P}_{\perp}^{3}\right) .
\end{gathered}
$$

This is the main result of this paper. Recall that $\zeta \equiv \frac{k_{y}^{+}}{p^{+}}=\frac{k_{\gamma \perp}}{p_{\perp}} e^{\eta_{k_{y}}-\eta_{p}}$ is the ratio of photon and quark energies in the final state, while $z$ is the fractional energy picked up by the photon from its parent quark. Our result applies when $1-z \ll 1$. Note that the gluon distributions in the expressions above are evaluated at the scale $(1-z) \tilde{P}_{\perp} / z$. Hence, these angular moments provide insight into the transverse momentum dependence of the gluon distributions of the target which is due to the fact that they involve another scale, i.e. the nonlinear ("saturation") scale $Q_{S}$. 


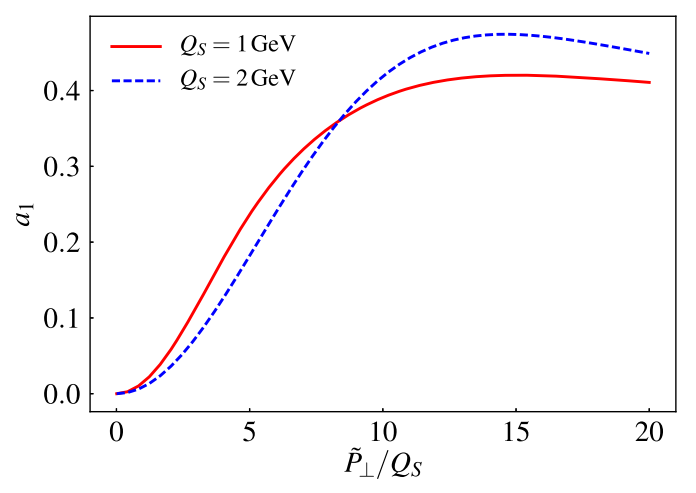

FIG. 3. $a_{1}$ for $g \rightarrow q \gamma$ to order $Q_{\perp}^{2} / \tilde{P}_{\perp}^{2}$ for the MV model with two different saturation scales. We take $Q_{\perp} / \tilde{P}_{\perp}=0.1, z=3 / 4$ and $\zeta=1$.

For the numerical results shown in Figs. 3 and 4 we have used $Q_{\perp} / \tilde{P}_{\perp}=0.1, z=3 / 4, \zeta=1$. Also, for simplicity we have computed the $F_{i}$ and $F_{i}^{(a, b)}$ gluon distributions in the McLerran-Venugopalan (MV) model [26] with detailed calculations in Appendix B, while leaving a numerical study of evolution effects for the future. Both $a_{1}\left(\tilde{P}_{\perp}\right)$ and $a_{2}\left(\tilde{P}_{\perp}\right)$ increase rapidly with transverse momentum and reach their maximal values approximately when $(1-z) \tilde{P}_{\perp} / z \approx 5 Q_{S}$. This maximum may disappear if evolution effects are taken into account, similar to their effect on the dipole scattering amplitude [27]. Also, we find that $a_{2}$ is substantially smaller than $a_{1}$ due to the suppression by one additional power of $Q_{\perp} / \tilde{P}_{\perp}$.

In Fig. 5 we plot $a_{1}$ as a function of the photon isolation cut $(1-z) / z$, which is the energy fraction carried by the final state quark collinear to the photon. The behavior of $a_{2}$ is very similar. As before we used $\zeta=1, Q_{\perp} / \tilde{P}_{\perp}=0.1$, $\tilde{P}_{\perp}=15 Q_{S}$, and we show curves for two different values of the saturation scale. It is interesting to observe how reducing $(1-z) / z$ increases the angular correlations.

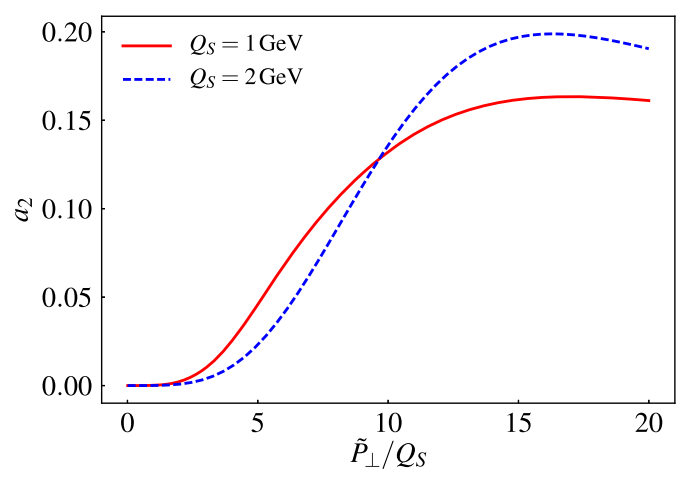

FIG. 4. $\quad a_{2}$ for $g \rightarrow q \gamma$ to order $Q_{\perp}^{2} / \tilde{P}_{\perp}^{2}$ for the MV model with two different saturation scales. We take $Q_{\perp} / \tilde{P}_{\perp}=0.1, z=3 / 4$ and $\zeta=1$.

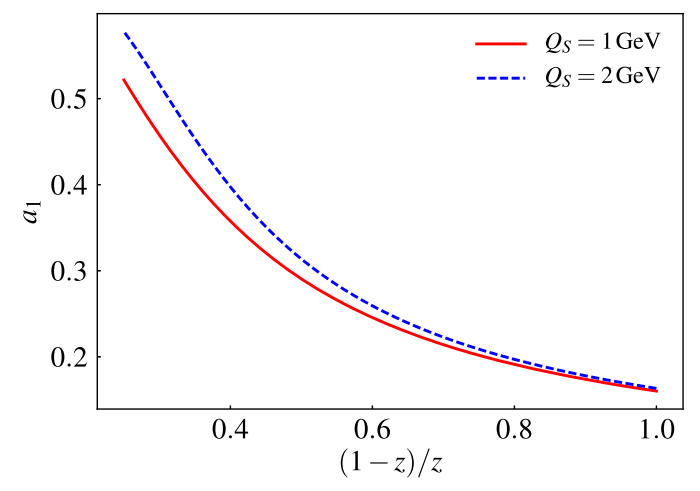

FIG. 5. $a_{1}$ for $g \rightarrow q \gamma$ to order $Q_{\perp}^{2} / \tilde{P}_{\perp}^{2}$ as a function of the photon isolation cut for the MV model with two different saturation scales. We take $Q_{\perp} / \tilde{P}_{\perp}=0.1, \tilde{P}_{\perp}=15 Q_{S}$ and $\zeta=1$.

We may also compute the "angular harmonics" given by

$v_{n}^{2} \equiv\langle\cos (n \Phi)\rangle \equiv \frac{\int_{0}^{2 \pi} d \phi \frac{d \sigma}{d^{2} \tilde{\boldsymbol{P}}_{\perp} d^{2} \boldsymbol{Q}_{\perp} d \eta_{p} d \eta_{k_{\gamma}} d z} \cos (n \Phi)}{\int_{0}^{2 \pi} d \phi \frac{d \sigma}{d^{2} \tilde{\boldsymbol{P}}_{\perp} d^{2} \boldsymbol{Q}_{\perp} d \eta_{p} d \eta_{k_{\gamma}} d z}}$,

where now $\Phi$ is the angle between $\boldsymbol{k}_{\gamma \perp}$ and $\boldsymbol{p}_{\perp}$. Hence, we evaluate these angular harmonics at fixed photon-jet transverse momentum imbalance $Q_{\perp}$ and average transverse momentum $\tilde{P}_{\perp}$ by averaging over their relative orientation. Because of the requirement that $Q_{\perp} \ll \tilde{P}_{\perp}$ the angle $\Phi$ is close to $\pm \pi$. Up to order $\left(Q_{\perp} / \tilde{P}_{\perp}\right)^{2}$ we have

$$
\begin{gathered}
\cos \Phi=-1+\frac{1}{4}\left(\frac{Q_{\perp}}{\tilde{P}_{\perp}}\right)^{2}(1-\cos 2 \phi), \\
\cos 2 \Phi=1-\left(\frac{Q_{\perp}}{\tilde{P}_{\perp}}\right)^{2}(1-\cos 2 \phi), \\
\cos 3 \Phi=-1+\frac{9}{4}\left(\frac{Q_{\perp}}{\tilde{P}_{\perp}}\right)^{2}(1-\cos 2 \phi) .
\end{gathered}
$$

Since $\langle\cos 2 \phi\rangle=a_{2}$ is itself of order $\left(Q_{\perp} / \tilde{P}_{\perp}\right)^{2}$ we have, to this order, the following predictions:

$$
\begin{aligned}
& v_{1}^{2} \equiv\langle\cos \Phi\rangle=-1+\frac{1}{4}\left(\frac{Q_{\perp}}{\tilde{P}_{\perp}}\right)^{2}=-1+\frac{\left|\boldsymbol{k}_{\gamma \perp}+\boldsymbol{p}_{\perp}\right|^{2}}{\left|\boldsymbol{k}_{\gamma \perp}-\boldsymbol{p}_{\perp}\right|^{2}}, \\
& v_{2}^{2} \equiv\langle\cos 2 \Phi\rangle=1-\left(\frac{Q_{\perp}}{\tilde{P}_{\perp}}\right)^{2}=1-4 \frac{\left|\boldsymbol{k}_{\gamma \perp}+\boldsymbol{p}_{\perp}\right|^{2}}{\left|\boldsymbol{k}_{\gamma \perp}-\boldsymbol{p}_{\perp}\right|^{2}}, \quad(66) \\
& v_{3}^{2} \equiv\langle\cos 3 \Phi\rangle=-1+\frac{9}{4}\left(\frac{Q_{\perp}}{\tilde{P}_{\perp}}\right)^{2}=-1+9 \frac{\left|\boldsymbol{k}_{\gamma \perp}+\boldsymbol{p}_{\perp}\right|^{2}}{\left|\boldsymbol{k}_{\gamma \perp}-\boldsymbol{p}_{\perp}\right|^{2}}
\end{aligned}
$$


These expressions provide the leading dependence of $v_{1}^{2}$, $v_{2}^{2}, v_{3}^{2}$ on $Q_{\perp} / \tilde{P}_{\perp}$. These moments are insensitive to the transverse momentum dependence of the gluon distributions $F_{i}$.

\section{SUMMARY AND DISCUSSION}

In this paper we have computed the photon-jet cross section corresponding to the process $g \rightarrow q \gamma$ in the small- $x$ regime of $p+A$ collisions. We focus on nearly back to back configurations with a photon-jet transverse momentum imbalance $\boldsymbol{Q}_{\perp}=\boldsymbol{k}_{\gamma \perp}+\boldsymbol{p}_{\perp}$ of much smaller magnitude than their average transverse momentum $\tilde{\boldsymbol{P}}_{\perp}=\left(\boldsymbol{p}_{\perp}-\boldsymbol{k}_{\gamma \perp}\right) / 2$. In this limit the cross section for the process can be expressed in terms of transversemomentum-dependent gluon distributions for the heavyion target. We determine which gluon distributions appear in this process and find, in particular, that the linearly polarized distributions do not enter (similar to $q \rightarrow q \gamma$ ). We also show that the contribution from the leading-order $q \rightarrow q \gamma$ process is suppressed by two powers of $Q_{\perp} / Q_{S}$ as compared to $g \rightarrow q \gamma$ if $Q_{\perp}<$ $Q_{S}$ is less than the saturation scale of the nucleus.

The calculation begins by considering scattering of a gluon from the projectile proton off the strong color field of the target ion, thereby producing a quark, an antiquark, and a photon. The $q \gamma$ final state is obtained by integrating over the quark from which the photon was emitted (in the collinear approximation). We find that the transverse momentum scale that appears in the gluon distributions of the target is given by the leftover transverse momentum $1-z$ of the quark relative to that of the photon, $z$, times the hard scale $\tilde{P}_{\perp}$. Hence, configurations where the photon picks up most of the momentum of the parent quark, $1-z \ll 1$, so that a second hadronic jet collinear to the photon is not observed (due to isolation cuts), do probe the gluon distributions of the target in the nonlinear regime.

Finally, we provide analytic expressions and qualitative numerical estimates for $a_{1}=\langle\cos \phi\rangle$ and $a_{2}=$ $\langle\cos 2 \phi\rangle$ angular moments, where $\phi$ denotes the angle between $\boldsymbol{Q}_{\perp}$ and $\tilde{\boldsymbol{P}}_{\perp}$. We predict that $a_{2} \ll a_{1}$ due to a power suppression by a factor of $Q_{\perp} / \tilde{P}_{\perp}$. The $\tilde{P}_{\perp}$ dependence of these angular correlations provides insight into the transverse momentum dependence of the gluon distributions of the target. In particular, numerically large $a_{1}$ and $a_{2}$ are obtained for more restrictive photon isolation cuts, and when the transverse momentum scale in the gluon distributions is on the order of a few times the saturation scale of the heavyion target.
We have also derived analytic estimates for the "azimuthal angular harmonics" $v_{n}^{2}=\langle\cos n \Phi\rangle$ up to $n=3$ and $\mathcal{O}\left(Q_{\perp}^{2} / \tilde{P}_{\perp}^{2}\right)$, where now $\Phi$ denotes the angle between the transverse momentum $\boldsymbol{k}_{\gamma \perp}$ of the photon and that of the jet, $\boldsymbol{p}_{\perp}$. However, up to order $\left(Q_{\perp} / \tilde{P}_{\perp}\right)^{2}$ these $v_{n}$ moments are insensitive to the transverse momentum dependence of the gluon distributions.

If photon-jet angular correlations in $p+A$ collisions can indeed be studied experimentally at high-energy colliders then a more quantitative evaluation of the angular distributions than presented in this initial study would be warranted. Most importantly, small- $x$ evolution effects on the transverse-momentum-dependent gluon distributions should be incorporated. This could be done by substituting the solution of the BK equation for $\Gamma_{x_{A}}\left(x_{\perp}^{2}\right)$ into the expressions for $F_{i}\left(x_{A}, k_{\perp}^{2}\right)$ given in Appendix B.

One should also account for the Sudakov suppression which arises due to the presence of the two scales $Q_{\perp}$ and $\tilde{P}_{\perp}[6,28]$. On the other hand, for $\tilde{P}_{\perp}$ only a few times greater than $Q_{\perp}$ (to suppress power corrections reasonably well) $\log \tilde{P}_{\perp} / Q_{\perp}$ is numerically not much greater than 1 and we would not expect a very strong effect on the angular distributions. In any case, the results presented here for the $g \rightarrow q \gamma$ process could be used as a starting point for such improvements. Our present analysis already suggests that photon-jet correlations in $p+A$ should provide valuable insight into transverse-momentum-dependent gluon distributions in the regime of nonlinear color fields.

\section{ACKNOWLEDGMENTS}

S. B. was supported by the European Union Seventh Framework Programme (FP7 2007-2013) under Grant No. 291823 and Marie Curie FP7-PEOPLE-2011COFUND New International Fellowship Mobility Programme for Experienced Researchers in Croatia (NEWFELPRO) Grant No. 48. S. B. also acknowledges the support of Hrvatska Zaklada Za Znanost (HRZZ) Grant No. 8799. A. D. gratefully acknowledges support by the Deparmtent of Energy (DOE) Office of Nuclear Physics through Grant No. DE-FG02-09ER41620 and from the City University of New York through the PSC-CUNY Research Grant No. 60262-0048.

\section{APPENDIX A: EXPANSION IN POWERS OF GLUON MOMENTA}

Here we perform an expansion of the amplitude for $g \rightarrow q \bar{q} \gamma$, given in (4), in powers of gluon momenta from the proton and from the nucleus. We start by generalizing the expressions for the matrices $R_{\beta}^{\mu}$ from Eqs. (9)-(13) as 


$$
\begin{aligned}
& R_{1}^{\mu \nu} \equiv-\frac{1}{P^{2}} \gamma^{\mu} \frac{\not w+m}{w^{2}-m^{2}} \gamma^{\nu} \\
& R_{2}^{\mu \nu} \equiv-\frac{1}{P^{2}} \gamma^{\nu} \frac{-\not p+m}{v^{2}-m^{2}} \gamma^{\mu}, \\
& R_{9}^{\mu \nu \alpha_{1} \alpha_{2}} \equiv(-i) \int_{-\infty}^{\infty} \frac{d k^{-}}{2 \pi} \tilde{R}_{9}^{\mu \nu \alpha_{1} \alpha_{2}}, \quad \tilde{R}_{9}^{\mu \nu \alpha_{1} \alpha_{2}} \equiv \gamma^{\mu} \frac{\not w+m}{w^{2}-m^{2}+i \epsilon} \gamma^{\alpha_{1}} \frac{\not h+m}{u^{2}-m^{2}+i \epsilon} \gamma^{\nu} \frac{\not+m}{l^{2}-m^{2}+i \epsilon} \gamma^{\alpha_{2}} \\
& R_{10}^{\mu \nu \alpha_{1} \alpha_{2}} \equiv(-i) \int_{-\infty}^{\infty} \frac{d k^{-}}{2 \pi} \tilde{R}_{10}^{\mu \nu \alpha_{1} \alpha_{2}}, \quad \tilde{R}_{10}^{\mu \nu \alpha_{1} \alpha_{2}} \equiv \gamma^{\alpha_{1}} \frac{\not g+m}{g^{2}-m^{2}+i \epsilon} \gamma^{\nu} \frac{\not h+m}{h^{2}-m^{2}+i \epsilon} \gamma^{\alpha_{2}} \frac{-\not h+m}{v^{2}-m^{2}+i \epsilon} \gamma^{\mu}, \\
& R_{11}^{\mu \nu \alpha_{1} \alpha_{2}} \equiv(-i) \int_{-\infty}^{\infty} \frac{d k^{-}}{2 \pi} \tilde{R}_{11}^{\mu \nu \alpha_{1} \alpha_{2}}, \quad \tilde{R}_{11}^{\mu \nu \alpha_{1} \alpha_{2}} \equiv \gamma^{\alpha_{1}} \frac{\not g+m}{g^{2}-m^{2}+i \epsilon} \gamma^{\mu} \frac{\not h+m}{u^{2}-m^{2}+i \epsilon} \gamma^{\nu} \frac{\not l+m}{l^{2}-m^{2}+i \epsilon} \gamma^{\alpha_{2}} \text {, } \\
& R_{12}^{\mu \nu \alpha_{1} \alpha_{2}} \equiv(-i) \int_{-\infty}^{\infty} \frac{d k^{-}}{2 \pi} \tilde{R}_{12}^{\mu \nu \alpha_{1} \alpha_{2}}, \quad \tilde{R}_{12}^{\mu \nu \alpha_{1} \alpha_{2}} \equiv \gamma^{\alpha_{1}} \frac{\not g+m}{g^{2}-m^{2}+i \epsilon} \gamma^{\nu} \frac{\not h+m}{h^{2}-m^{2}+i \epsilon} \gamma^{\mu} \frac{\not l+m}{l^{2}-m^{2}+i \epsilon} \gamma^{\alpha_{2}} \text {, }
\end{aligned}
$$

and also

$$
R_{g}^{\mu \nu}=\sum_{\beta=1}^{2} R_{\beta}^{\mu \nu}, \quad R_{q \bar{q}}^{\mu \nu \alpha_{1} \alpha_{2}}=\sum_{\beta=9}^{12} R_{\beta}^{\mu \nu \alpha_{1} \alpha_{2}} .
$$

Performing the $k^{-}$integration in $R_{\beta}^{\mu \nu \alpha_{1} \alpha_{2}}, \beta=9, \ldots, 12$, we can show that $T_{q \bar{q}}^{\mu}=R_{q \bar{q}}^{\mu-++}$ and obviously $T_{g}^{\mu}=R_{g}^{\mu \nu} C_{L \nu}$.

Contracting $R_{\beta}^{\mu \nu++}$ for $\beta=9, \ldots, 12$ with $k_{1 \nu}$ we get

$$
\begin{aligned}
& k_{1 \nu} R_{9}^{\mu \nu++}=-\frac{\gamma^{\mu}(\not w+m) \gamma^{+}}{w^{2}-m^{2}}=P^{2} R_{1}^{\mu+}, \\
& k_{1 \nu} R_{10}^{\mu \nu++}=-\frac{\gamma^{+}(-\not p+m) \gamma^{\mu}}{v^{2}-m^{2}}=P^{2} R_{2}^{\mu+}, \\
& k_{1 \nu} R_{11}^{\mu \nu++}=-\frac{\gamma^{+}(\not \partial+m) \gamma^{\mu}(\not l+m) \gamma^{+}}{S}=-k_{1 \nu} R_{12}^{\mu \nu++} .
\end{aligned}
$$

Decomposing these expressions we can use $k_{1}^{+} R_{q \bar{q}}^{\mu-++}=P^{+} R_{q \bar{q}}^{\mu-++}$ to redefine $T_{g}^{\mu}$. We can write the amplitude as

$$
\begin{aligned}
\mathcal{M}^{\mu}\left(\boldsymbol{p}, \boldsymbol{q}, \boldsymbol{k}_{\gamma}\right)= & -q_{f} e g^{2} \int_{\boldsymbol{k}_{\perp} \boldsymbol{k}_{\perp \perp}} \int_{\boldsymbol{x}_{\perp} \boldsymbol{y}_{\perp}} \frac{\rho_{p}^{a}\left(\boldsymbol{k}_{1 \perp}\right)}{k_{1 \perp}^{2}} e^{i \boldsymbol{k}_{\perp} \cdot \boldsymbol{x}_{\perp}+i\left(\boldsymbol{k}_{\perp \perp}-\boldsymbol{k}_{\perp}\right) \cdot \boldsymbol{y}_{\perp}} \\
& \times \bar{u}(\boldsymbol{q})\left\{R_{g}^{\mu \nu} C_{\nu}\left(P, \boldsymbol{k}_{1 \perp}\right) U\left(\boldsymbol{x}_{\perp}\right)^{b a} t^{b}-\frac{k_{1 i}}{P^{+}} R_{q \bar{q}}^{\mu i++} \tilde{U}\left(\boldsymbol{x}_{\perp}\right) t^{a} \tilde{U}^{\dagger}\left(\boldsymbol{y}_{\perp}\right)\right\} v(\boldsymbol{p}),
\end{aligned}
$$

with

$$
C^{+}\left(q, \boldsymbol{k}_{1 \perp}\right)=0, \quad C^{-}\left(q, \boldsymbol{k}_{1 \perp}\right)=\frac{-2 \boldsymbol{k}_{1 \perp} \cdot\left(\boldsymbol{q}_{\perp}-\boldsymbol{k}_{1 \perp}\right)}{q^{+}+i \epsilon}, \quad \boldsymbol{C}_{\perp}\left(q ; \boldsymbol{k}_{1 \perp}\right)=\frac{\boldsymbol{q}_{\perp} k_{1 \perp}^{2}}{\left(q^{+}+i \epsilon\right)\left(q^{-}+i \epsilon\right)}-2 \boldsymbol{k}_{1 \perp} .
$$

Contracting $\tilde{R}_{\beta}^{\mu \nu \alpha_{1} \alpha_{2}}$ for $\beta=9, \ldots, 12$ with $k_{\alpha_{1}}$ or with $\left(k_{2}-k\right)_{\alpha_{2}}$ we get

$$
\begin{aligned}
& k_{\alpha_{1}} \tilde{R}_{9}^{\mu \nu \alpha_{1} \alpha_{2}}=-\gamma^{\mu} \frac{\not h+m}{w^{2}-m^{2}+i \epsilon} \gamma^{\nu} \frac{\not l+m}{l^{2}-m^{2}+i \epsilon} \gamma^{\alpha_{2}}+\gamma^{\mu} \frac{\not h+m}{u^{2}-m^{2}+i \epsilon} \gamma^{\nu} \frac{\not l+m}{l^{2}-m^{2}+i \epsilon} \gamma^{\alpha_{2}}, \\
& k_{\alpha_{1}} \tilde{R}_{10}^{\mu \nu \alpha_{1} \alpha_{2}}=-\gamma^{\nu} \frac{\not h+m}{h^{2}-m^{2}+i \epsilon} \gamma^{\alpha_{2}} \frac{-\not p+m}{v^{2}-m^{2}+i \epsilon} \gamma^{\mu}, \\
& k_{\alpha_{1}} \tilde{R}_{11}^{\mu \nu \alpha_{1} \alpha_{2}}=-\gamma^{\mu} \frac{\not h+m}{u^{2}-m^{2}+i \epsilon} \gamma^{\nu} \frac{\not l+m}{l^{2}-m^{2}+i \epsilon} \gamma^{\alpha_{2}}, \\
& k_{\alpha_{1}} \tilde{R}_{12}^{\mu \nu \alpha_{1} \alpha_{2}}=-\gamma^{\nu} \frac{\not h+m}{h^{2}-m^{2}+i \epsilon} \gamma^{\mu} \frac{\not l+m}{l^{2}-m^{2}+i \epsilon} \gamma^{\alpha_{2}} .
\end{aligned}
$$




$$
\begin{aligned}
& \left(k_{2}-k\right)_{\alpha_{2}} \tilde{R}_{9}^{\mu \nu \alpha_{1} \alpha_{2}}=\gamma^{\mu} \frac{\not w+m}{w^{2}-m^{2}+i \epsilon} \gamma^{\alpha_{1}} \frac{\not h+m}{u^{2}-m^{2}+i \epsilon} \gamma^{\nu}, \\
& \left(k_{2}-k\right)_{\alpha_{2}} \tilde{R}_{10}^{\mu \nu \alpha_{1} \alpha_{2}}=\gamma^{\alpha_{1}} \frac{\not g+m}{g^{2}-m^{2}+i \epsilon} \gamma^{\nu} \frac{-\not p+m}{v^{2}-m^{2}+i \epsilon} \gamma^{\mu}-\gamma^{\alpha_{1}} \frac{\not g+m}{g^{2}-m^{2}+i \epsilon} \gamma^{\nu} \frac{\not h+m}{h^{2}-m^{2}+i \epsilon} \gamma^{\mu}, \\
& \left(k_{2}-k\right)_{\alpha_{2}} \tilde{R}_{11}^{\mu \nu \nu \alpha_{1} \alpha_{2}}=\gamma^{\alpha_{1}} \frac{\not g+m}{g^{2}-m^{2}+i \epsilon} \gamma^{\mu} \frac{\not h+m}{u^{2}-m^{2}+i \epsilon} \gamma^{\nu}, \\
& \left(k_{2}-k\right)_{\alpha_{2}} \tilde{R}_{12}^{\mu \nu \alpha_{1} \alpha_{2}}=\gamma^{\alpha_{1}} \frac{\not g+m}{g^{2}-m^{2}+i \epsilon} \gamma^{\nu} \frac{\not h+m}{h^{2}-m^{2}+i \epsilon} \gamma^{\mu} .
\end{aligned}
$$

Next, we use Eq. (A6) to combine $\tilde{R}_{9}^{\mu \nu \alpha_{1} \alpha_{2}}$ with $\tilde{R}_{11}^{\mu \nu \alpha_{1} \alpha_{2}}$,

$$
k^{-}\left(\tilde{R}_{9}^{\mu \nu++}+\tilde{R}_{11}^{\mu \nu++}\right)=-k_{i_{1}}\left(\tilde{R}_{9}^{\mu \nu i_{1}+}+\tilde{R}_{11}^{\mu \nu i_{1}+}\right)-\gamma^{\mu} \frac{\not w+m}{w^{2}-m^{2}+i \epsilon} \gamma^{\nu} \frac{\not l+m}{l^{2}-m^{2}+i \epsilon} \gamma^{+},
$$

and we use (A7) to combine $\tilde{R}_{10}^{\mu \nu \alpha_{1} \alpha_{2}}$ with $\tilde{R}_{12}^{\mu \nu \alpha_{1} \alpha_{2}}$,

$$
\left(k_{2}-k\right)^{-}\left(\tilde{R}_{10}^{\mu \nu++}+\tilde{R}_{12}^{\mu \nu++}\right)=-\left(k_{2}-k\right)_{i_{2}}\left(\tilde{R}_{10}^{\mu \nu+i_{2}}+\tilde{R}_{12}^{\mu \nu+i_{2}}\right)+\gamma^{+} \frac{\not b+m}{g^{2}-m^{2}+i \epsilon} \gamma^{\nu} \frac{-\not p+m}{v^{2}-m^{2}+i \epsilon} \gamma^{\mu} .
$$

We first integrate these expressions over $k^{-}$. On the left-hand side we get $R_{9}^{\mu \nu++}+R_{11}^{\mu \nu++}$ and $R_{10}^{\mu \nu++}+R_{12}^{\mu \nu++}$, respectively. Taking the limit $k_{1 \perp}, k_{2 \perp}, k_{\perp} \rightarrow 0$ we find that in Eq. (A8) $k^{-}=P^{-}$, while in Eq. (A9) $k^{-}=0$. Combining these results together we get the following expression for $R_{q \bar{q}}^{\mu \nu++}$,

$$
\begin{aligned}
R_{q \bar{q}}^{\mu \nu++}= & \gamma^{\mu} \frac{\not p+m}{w^{2}-m^{2}} \frac{\not k_{\perp}}{P^{-}} \frac{\not h+m}{u^{2}-m^{2}} \gamma^{\nu}+\frac{\not \ell_{\perp}}{P^{-}} \frac{\not g+m}{g^{2}-m^{2}} \gamma^{\mu} \frac{\not h+m}{u^{2}-m^{2}} \gamma^{\nu}+\frac{\not k_{\perp}}{P^{-}} \frac{\not g+m}{g^{2}-m^{2}} \gamma^{\nu} \frac{-\not p+m}{v^{2}-m^{2}} \gamma^{\mu} \\
& -\gamma^{\nu} \frac{\not h+m}{h^{2}-m^{2}} \frac{\not k_{2 \perp}-\not k_{\perp}}{P^{-}} \frac{-\not p+m}{v^{2}-m^{2}} \gamma^{\mu}-\gamma^{\nu} \frac{\not h+m}{h^{2}-m^{2}} \gamma^{\mu} \frac{\not l+m}{l^{2}-m^{2}} \frac{\not k_{2 \perp}-\not k_{\perp}}{P^{-}}-\gamma^{\mu} \frac{\not w+m}{w^{2}-m^{2}} \gamma^{\nu} \frac{\not l+m \not k_{2 \perp}-\not k_{\perp}}{l^{2}-m^{2}} \\
& +\frac{1}{P^{-}} \gamma^{\mu} \frac{\not w+m}{w^{2}-m^{2}} \gamma^{\nu}+\frac{1}{P^{-}} \gamma^{\nu} \frac{-\not p+m}{v^{2}-m^{2}} \gamma^{\mu},
\end{aligned}
$$

where the first line comes from Eq. (A8) and so we should understand $k^{-}=P^{-}$, while the second line comes from Eq. (A9) and so $k^{-}=0$. The third line can be joined to $R_{g}^{\mu \nu}$ [defined in Eq. (A2)] which leads us to the final expression for the amplitude given in Eqs. (14)-(16).

\section{APPENDIX B: DISTRIBUTION FUNCTIONS}

We can write more explicit expressions for the distribution functions $F_{i}$ and $H_{i}$ by using the large $N_{c}$ expression for the four Wilson line correlator $C\left(x_{A}, \boldsymbol{x}_{\perp}, \boldsymbol{y}_{\perp}, \boldsymbol{y}_{\perp}^{\prime}, \boldsymbol{x}_{\perp}^{\prime}\right)$ in a Gaussian model [29,30],

$C\left(x_{A}, \boldsymbol{x}_{\perp}, \boldsymbol{y}_{\perp}, \boldsymbol{y}_{\perp}^{\prime}, \boldsymbol{x}_{\perp}^{\prime}\right)=\frac{N_{c}^{2}}{2} S\left(\left(\boldsymbol{x}_{\perp}-\boldsymbol{x}_{\perp}^{\prime}\right)^{2}\right) S\left(\left(\boldsymbol{y}_{\perp}-\boldsymbol{y}_{\perp}^{\prime}\right)^{2}\right)$,

where $S\left(x_{\perp}^{2}\right)=e^{-\Gamma\left(x_{\perp}^{2}\right)}$ is the $S$-matrix for a fundamental dipole of size $x_{\perp}$. Within the MV model [26] (without small$x$ evolution) we have

$$
\begin{aligned}
\Gamma\left(x_{\perp}^{2}\right) & =Q_{S}^{2} \int_{\boldsymbol{y}_{\perp}}\left[G_{0}\left(\boldsymbol{y}_{\perp}\right)-G_{0}\left(\boldsymbol{y}_{\perp}-\boldsymbol{x}_{\perp}\right)\right]^{2}, \\
Q_{S}^{2} & \equiv \frac{g^{4}\left(N_{c}^{2}-1\right)}{4 \pi N_{c}} \mu_{A}^{2},
\end{aligned}
$$

where $\mu_{A}^{2}$ is the conventional MV model parameter, i.e. the average valence color charge density squared per unit transverse area; $Q_{S}^{2}$ is the saturation scale; and $G_{0}\left(\boldsymbol{x}_{\perp}\right)$ is a solution of $\partial_{\perp}^{2} G_{0}\left(x_{\perp}\right)=\delta^{(2)}\left(x_{\perp}\right)$. Regularizing the IR divergence in the Fourier transform of $G_{0}\left(\boldsymbol{x}_{\perp}\right)$ as $G_{0}\left(\boldsymbol{k}_{\perp}\right)=$ $\left(\boldsymbol{k}_{\perp}^{2}+\Lambda^{2}\right)^{-1}$ we can find

$$
\Gamma\left(x_{\perp}^{2}\right)=\frac{Q_{S}^{2}}{2 \Lambda^{2}}\left[1-\left(x_{\perp} \Lambda\right) K_{1}\left(x_{\perp} \Lambda\right)\right],
$$

where $K_{1}(x)$ is the modified Bessel function of the second kind. Expanding around $x_{\perp} \Lambda \rightarrow 0$ we find

$$
\begin{aligned}
\Gamma\left(x_{\perp}^{2}\right) & =\frac{x_{\perp}^{2} Q_{S}^{2}}{8}\left(1-2 \gamma_{E}+\log 4-\log \frac{1}{x_{\perp}^{2} \Lambda^{2}}\right) \\
& =\frac{x_{\perp}^{2} Q_{S}^{2}}{8} \log \left(\frac{1}{x_{\perp}^{2} \Lambda_{\mathrm{IR}}^{2}}\right), \quad \Lambda_{\mathrm{IR}}^{2} \equiv \frac{\Lambda^{2}}{4 e^{1-2 \gamma_{E}}} .
\end{aligned}
$$

However, Eq. (B1) is not restricted to the MV model. One can include small- $x$ evolution effects by solving the BK equation [21] for $\Gamma\left(x_{\perp}^{2}\right)$ which now becomes also a function of $x_{A}: \Gamma\left(x_{\perp}^{2}\right) \rightarrow \Gamma_{x_{A}}\left(x_{\perp}^{2}\right)$. We proceed with this more general formulation and express the distribution functions in terms of $\Gamma_{x_{A}}\left(x_{\perp}^{2}\right)$. We find 


$$
\begin{gathered}
F_{1}\left(x_{A}, k_{2 \perp}^{2}\right)=\left(\pi R_{A}^{2}\right) 4 \pi N_{c}^{2} \int_{0}^{\infty} x_{\perp} d x_{\perp} J_{0}\left(k_{2 \perp} x_{\perp}\right)\left\{\Gamma_{x_{A}}^{(1)}\left(x_{\perp}^{2}\right)+x_{\perp}^{2}\left[\Gamma_{x_{A}}^{(2)}\left(x_{\perp}^{2}\right)-\left(\Gamma_{x_{A}}^{(1)}\left(x_{\perp}^{2}\right)\right)^{2}\right]\right\} e^{-2 \Gamma_{x_{A}}\left(x_{\perp}^{2}\right)} \\
F_{2}\left(x_{A}, k_{2 \perp}^{2}\right)=-\left(\pi R_{A}^{2}\right) 4 \pi N_{c}^{2} \int_{0}^{\infty} x_{\perp} d x_{\perp} J_{0}\left(k_{2 \perp} x_{\perp}\right) x_{\perp}^{2}\left(\Gamma_{x_{A}}^{(1)}\left(x_{\perp}^{2}\right)\right)^{2} e^{-2 \Gamma_{x_{A}}\left(x_{\perp}^{2}\right)} \\
F_{3}\left(x_{A}, k_{2 \perp}^{2}\right)=\left(\pi R_{A}^{2}\right) 4 \pi N_{c}^{2} \int_{0}^{\infty} x_{\perp} d x_{\perp} J_{0}\left(k_{2 \perp} x_{\perp}\right)\left\{\Gamma_{x_{A}}^{(1)}\left(x_{\perp}^{2}\right)+x_{\perp}^{2}\left[\Gamma_{x_{A}}^{(2)}\left(x_{\perp}^{2}\right)-2\left(\Gamma_{x_{A}}^{(1)}\left(x_{\perp}^{2}\right)\right)^{2}\right]\right\} e^{-2 \Gamma_{x_{A}}\left(x_{\perp}^{2}\right)} \\
H_{1}\left(x_{A}, k_{2 \perp}^{2}\right)=-\left(\pi R_{A}^{2}\right) 4 \pi N_{c}^{2} \int_{0}^{\infty} x_{\perp} d x_{\perp} J_{2}\left(k_{2 \perp} x_{\perp}\right) x_{\perp}^{2}\left[\Gamma_{x_{A}}^{(2)}\left(x_{\perp}^{2}\right)-\left(\Gamma_{x_{A}}^{(1)}\left(x_{\perp}^{2}\right)\right)^{2}\right] e^{-2 \Gamma_{x_{A}}\left(x_{\perp}^{2}\right)} \\
H_{2}\left(x_{A}, k_{2 \perp}^{2}\right)=\left(\pi R_{A}^{2}\right) 4 \pi N_{c}^{2} \int_{0}^{\infty} x_{\perp} d x_{\perp} J_{2}\left(k_{2 \perp} x_{\perp}\right) x_{\perp}^{2}\left(\Gamma_{x_{A}}^{(1)}\left(x_{\perp}^{2}\right)\right)^{2} e^{-2 \Gamma_{x_{A}}\left(x_{\perp}^{2}\right)} \\
H_{3}\left(x_{A}, k_{2 \perp}^{2}\right)=-\left(\pi R_{A}^{2}\right) 4 \pi N_{c}^{2} \int_{0}^{\infty} x_{\perp} d x_{\perp} J_{2}\left(k_{2 \perp} x_{\perp}\right) x_{\perp}^{2}\left[\Gamma_{x_{A}}^{(2)}\left(x_{\perp}^{2}\right)-2\left(\Gamma_{x_{A}}^{(1)}\left(x_{\perp}^{2}\right)\right)^{2}\right] e^{-2 \Gamma_{x_{A}}\left(x_{\perp}^{2}\right)}
\end{gathered}
$$

where $\Gamma_{x_{A}}^{(n)}\left(x_{\perp}^{2}\right) \equiv d^{n} \Gamma_{x_{A}}\left(x_{\perp}^{2}\right) / d\left(x_{\perp}^{2}\right)^{n}$. In the MV model the $F$-functions become

$$
\begin{aligned}
& F_{1}\left(x_{A}, k_{2 \perp}^{2}\right)=\left(\pi R_{A}^{2}\right) \frac{Q_{S}^{2} N_{c}^{2}}{16} \int_{0}^{\infty} x_{\perp} d x_{\perp} J_{0}\left(k_{2 \perp} x_{\perp}\right)\left(8 \pi \log \frac{1}{x_{\perp}^{2} \Lambda_{\mathrm{IR}}^{2}}-\pi x_{\perp}^{2} Q_{S}^{2} \log ^{2} \frac{1}{x_{\perp}^{2} \Lambda_{\mathrm{IR}}^{2}}\right) e^{-\frac{Q_{S}^{2} x_{\perp}^{2}}{4} \log \frac{1}{x_{\perp}^{2} \Lambda_{\mathrm{IR}}^{2}}} \\
& F_{2}\left(x_{A}, k_{2 \perp}^{2}\right)=\left(\pi R_{A}^{2}\right) \frac{\pi Q_{S}^{2} N_{c}^{2}}{8} \int_{0}^{\infty} x_{\perp} d x_{\perp} J_{0}\left(k_{2 \perp} x_{\perp}\right) x_{\perp}^{2} Q_{S}^{2} \log ^{2} \frac{1}{x_{\perp}^{2} \Lambda_{\mathrm{IR}}^{2}} e^{-\frac{Q_{S}^{2} x_{\perp}^{2}}{4} \log _{\frac{1}{x_{\perp}^{2} \Lambda_{\mathrm{IR}}^{2}}}} \\
& F_{3}\left(x_{A}, k_{2 \perp}^{2}\right)=\left(\pi R_{A}^{2}\right) \frac{Q_{S}^{2} N_{c}^{2}}{8} \int_{0}^{\infty} x_{\perp} d x_{\perp} J_{0}\left(k_{2 \perp} x_{\perp}\right)\left(4 \pi \log \frac{1}{x_{\perp}^{2} \Lambda_{\mathrm{IR}}^{2}}-\pi Q_{S}^{2} x_{\perp}^{2} \log ^{2} \frac{1}{x_{\perp}^{2} \Lambda_{\mathrm{IR}}^{2}}\right) e^{-\frac{Q_{S}^{2} x_{\perp}^{2}}{4} \log _{\frac{1}{x_{\perp}^{2} \Lambda_{\mathrm{IR}}^{2}}}}
\end{aligned}
$$

[1] J. Jalilian-Marian, Nucl. Phys. A770, 210 (2006); Eur. Phys. J. C 61, 789 (2009); J. Jalilian-Marian and A. H. Rezaeian, Phys. Rev. D 86, 034016 (2012).

[2] B. Z. Kopeliovich, A. V. Tarasov, and A. Schäfer, Phys. Rev. C 59, 1609 (1999); B. Z. Kopeliovich, H. J. Pirner, A. H. Rezaeian, and I. Schmidt, Phys. Rev. D 77, 034011 (2008).

[3] F. Gelis and J. Jalilian-Marian, Phys. Rev. D 66, 014021 (2002).

[4] R. Baier, A. H. Mueller, and D. Schiff, Nucl. Phys. A741, 358 (2004).

[5] F. Dominguez, J. W. Qiu, B. W. Xiao, and F. Yuan, Phys. Rev. D 85, 045003 (2012).

[6] D. Boer, P. J. Mulders, J. Zhou, and Y.j. Zhou, J. High Energy Phys. 10 (2017) 196.

[7] S. Benic, K. Fukushima, O. Garcia-Montero, and R. Venugopalan, J. High Energy Phys. 01 (2017) 115.

[8] C. J. Bomhof, P. J. Mulders, and F. Pijlman, Eur. Phys. J. C 47, 147 (2006).

[9] F. Dominguez, C. Marquet, B. W. Xiao, and F. Yuan, Phys. Rev. D 83, 105005 (2011).

[10] E. Akcakaya, A. Schäfer, and J. Zhou, Phys. Rev. D 87, 054010 (2013).

[11] Yu. V. Kovchegov and E. Levin, Quantum Chromodynamics at High Energy, Cambridge Monographs 33 (Cambridge Univ. Press, Cambridge, England, 2012).
[12] T. Pietrycki and A. Szczurek, Phys. Rev. D 76, 034003 (2007); A. V. Lipatov and N. P. Zotov, Phys. Rev. D 90, 094005 (2014).

[13] T. Jezo, M. Klasen, and F. Knig, J. High Energy Phys. 11 (2016) 033.

[14] M. Klasen, C. Klein-Bösing, and H. Poppenborg, arXiv: 1709.04154.

[15] D. Boer, P. J. Mulders, and C. Pisano, Phys. Lett. B 660, 360 (2008).

[16] A. Adare et al. (PHENIX Collaboration), Phys. Rev. C 80, 024908 (2009); Phys. Rev. D 82, 072001 (2010); Phys. Rev. Lett. 111, 032301 (2013); Phys. Rev. D 95, 072002 (2017).

[17] L. Adamczyk et al. (STAR Collaboration), Phys. Rev. C 96, 024905 (2017); A. Schmah et al. (STAR Collaboration), Nucl. Phys. A 967, 11 (2017); N. R. Sahoo et al. (STAR Collaboration), Nucl. Phys. A 967, 480 (2017);

[18] J. L. Albacete, N. Armesto, J. G. Milhano, and C. A. Salgado, Phys. Rev. D 80, 034031 (2009); J. L. Albacete, N. Armesto, J. G. Milhano, P. Quiroga-Arias, and C. A. Salgado, Eur. Phys. J. C 71, 1705 (2011); J. L. Albacete, J. G. Milhano, P. Quiroga-Arias, and J. Rojo, Eur. Phys. J. C 72, 2131 (2012).

[19] G. Aad et al. (ATLAS Collaboration), Nucl. Phys. B875, 483 (2013). 
[20] CMS Collaboration, Report No. CMS-PAS-HIN-13-006.

[21] I. Balitsky, Nucl. Phys. B463, 99 (1996); Y. V. Kovchegov, Phys. Rev. D 60, 034008 (1999).

[22] C. Marquet, C. Roiesnel, and P. Taels, arXiv:1710.05698.

[23] S. Frixione, Phys. Lett. B 429, 369 (1998).

[24] A. Dumitru and J. Jalilian-Marian, Phys. Lett. B 547, 15 (2002); A. Dumitru, A. Hayashigaki, and J. Jalilian-Marian, Nucl. Phys. A765, 464 (2006).

[25] L. N. Lipatov, Yad. Fiz. 23, 642 (1976) [Sov. J. Nucl. Phys. 23, 338 (1976)]; E. A. Kuraev, L. N. Lipatov, and V. S. Fadin, Zh. Eksp. Teor. Fiz. 72, 377 (1977) [Sov. Phys. JETP 45, 199 (1977)]; I. I. Balitsky and L. N. Lipatov, Yad. Fiz. 28, 1597 (1978) [Sov. J. Nucl. Phys. 28, 822 (1978)].
[26] L. D. McLerran and R. Venugopalan, Phys. Rev. D 49, 2233 (1994); 49, 3352 (1994); Yu. V. Kovchegov, Phys. Rev. D 54, 5463 (1996).

[27] J. L. Albacete, N. Armesto, A. Kovner, C. A. Salgado, and U.A. Wiedemann, Phys. Rev. Lett. 92, 082001 (2004).

[28] A. H. Mueller, B. W. Xiao, and F. Yuan, Phys. Rev. D 88, 114010 (2013); L. Zheng, E. C. Aschenauer, J. H. Lee, and B. W. Xiao, Phys. Rev. D 89, 074037 (2014).

[29] J. P. Blaizot, F. Gelis, and R. Venugopalan, Nucl. Phys. A743, 57 (2004).

[30] K. Fukushima and Y. Hidaka, J. High Energy Phys. 06 (2007) 040. 